\title{
RANDOM CONDUCTANCE MODELS WITH STABLE-LIKE JUMPS: HEAT KERNEL ESTIMATES AND HARNACK INEQUALITIES
}

\author{
XIN CHEN TAKASHI KUMAGAI JIAN WANG
}

\begin{abstract}
We establish two-sided heat kernel estimates for random conductance models with non-uniformly elliptic (possibly degenerate) stable-like jumps on graphs. These are long range counterparts of well known two-sided Gaussian heat kernel estimates by M.T. Barlow for nearest neighbor (short range) random walks on the supercritical percolation cluster. Unlike the cases for nearest neighbor conductance models, the idea through parabolic Harnack inequalities does not work, since even elliptic Harnack inequalities do not hold in the present setting. As an application, we establish the local limit theorem for the models.
\end{abstract}

Keywords: conductance models with non-uniformly elliptic stable-like jumps; heat kernel estimate; Harnack inequality; Dynkin-Hunt formula

MSC 2010: 60G51; 60G52; 60J25; 60J75.

\section{INTRODUCTION}

Consider a bond percolation on $\mathbb{Z}^{d}, d \geqslant 2$; namely, on each nearest neighbor bond $x, y \in \mathbb{Z}^{d}$ with $|x-y|=1$, we put a random conductance $w_{x, y}$ in such a way that $\left\{w_{x, y}(\omega): x, y \in \mathbb{Z}^{d},|x-y|=1\right\}$ are i.i.d. Bernoulli so that $\mathbb{P}\left(w_{x, y}(\omega)=1\right)=p$ and $\mathbb{P}\left(w_{x, y}(\omega)=0\right)=1-p$ for some $p \in[0,1]$. It is known that there exists a constant $p_{c}\left(\mathbb{Z}^{d}\right) \in(0,1)$ such that almost surely there exists a unique infinite cluster $\mathcal{C}_{\infty}(\omega)$ (i.e. a connected component of bonds with conductance 1) when $p>p_{c}\left(\mathbb{Z}^{d}\right)$ and no infinite cluster when $p<p_{c}\left(\mathbb{Z}^{d}\right)$. Suppose $p>p_{c}\left(\mathbb{Z}^{d}\right)$ and consider a continuous time simple random walk $\left(X_{t}^{\omega}\right)_{t \geqslant 0}$ on the infinite cluster. Let $p^{\omega}(t, x, y)$ be the heat kernel (or the transition density function) of $X^{\omega}$, i.e.,

$$
p^{\omega}(t, x, y):=\frac{\mathbb{P}^{x}\left(X_{t}^{\omega}=y\right)}{\mu_{y}},
$$

where $\mu_{y}$ is a number of bonds whose one end is $y$. In the cerebrated paper [8], Barlow proved the following detailed heat kernel estimates those are almost sure w.r.t. the randomness of the environment; namely the following quenched estimates:

There exist random variables $\left\{R_{x}(\omega)\right\}_{x \in \mathbb{Z}^{d}}$ with $R_{x}(\omega) \in[1, \infty)$ for all $x \in \mathcal{C}_{\infty}(\omega)$ $\mathbb{P}$-a.s. $\omega$ and constants $c_{i}=c_{i}(d, p), i=1, \cdots, 4$ such that for all $x, y \in \mathcal{C}_{\infty}(\omega)$ with $t \geqslant|x-y| \vee R_{x}(\omega), p(t, x, y)$ satisfies the following

$$
c_{1} t^{-d / 2} \exp \left(-c_{2}|x-y|^{2} / t\right) \leqslant p^{\omega}(t, x, y) \leqslant c_{3} t^{-d / 2} \exp \left(-c_{4}|x-y|^{2} / t\right) .
$$

Note that because of the degenerate structure of the (random) environment, we cannot expect (1.1) to hold for all $t \geqslant 1$. Barlow's results assert that such a Gaussian estimate holds as a long time estimate despite of the degenerate structure.

X. Chen: Department of Mathematics, Shanghai Jiao Tong University, 200240 Shanghai, P.R. China. chenxin217@sjtu.edu.cn.

T. Kumagai: Research Institute for Mathematical Sciences, Kyoto University, Kyoto 606-8502, Japan. kumagai@kurims.kyoto-u.ac.jp.

J. Wang: College of Mathematics and Informatics \& Fujian Key Laboratory of Mathematical Analysis and Applications, Fujian Normal University, 350007 Fuzhou, P.R. China. jianwang@fjnu.edu.cn. 
When the conductances are bounded from above and below (the uniformly elliptic case) and the global volume doubling condition holds, it is well known that the associated heat kernel for the nearest neighbor conductance models obeys two-sided Gaussian estimates (see e.g. [31, 36]). When the conductances are non-uniformly elliptic, the situation becomes complex and delicate. The supercritical bond percolation discussed above is a typical example. (We note that Mathieu and Remy ([49]) also obtained a large time on-diagonal heat kernel upper bound for this model.)

Barlow's results have many applications. For example, they were applied crucially in the proofs of quenched local central limit theorem and quenched invariant principle for the model. The results have been extended to nearest neighbor conductance models with ergodic media in [4, 53], and these large time heat kernel estimates (and parabolic Harnack inequalities) have been key estimates in the field of random conductance models, see [1, 2, $3,4,5,6,10,11,16,22,20,21,50,51,54,52,53]$ or $[19,46]$ for the survey on these topics.

In this paper, we consider quenched heat kernel estimates for random conductance models that allow big jumps. In particular, we establish two-sided heat kernel estimates for random conductance models with non-uniformly elliptic and possibly degenerate stable-like jumps on graphs. Despite of the fundamental importance of the problem, so far there are only a few results for conductance models with long range jumps. As far as we are aware, this is the first work on detailed heat kernel estimates for possibly degenerate random walks with long range jumps. We now explain our framework and a result.

Suppose that $G=\left(V, E_{V}\right)$ is a locally finite connected infinite graph, where $V$ and $E_{V}$ denote the collection of vertices and edges respectively. For $x \neq y \in V$, we write $\rho(x, y)$ for the graph distance, i.e., $\rho(x, y)$ is the smallest positive length of a path (that is, a sequence $x_{0}=x, x_{1}, \cdots, x_{l}=y$ such that $\left(x_{i}, x_{i+1}\right) \in E_{V}$ for all $\left.0 \leqslant i \leqslant l-1\right)$ joining $x$ and $y$. We set $\rho(x, x)=0$ for all $x \in V$. Let $B(x, r):=\{y \in V: \rho(y, x) \leqslant r\}$ denote the ball with center $x \in V$ and radius $r \geqslant 1$. Let $\mu$ be a measure on $V$ such that the following assumption holds.

Assumption ( $d$-Vol). There are constants $R_{0} \geqslant 1, \kappa>0, c_{\mu} \geqslant 1, \theta \in(0,1)$ and $d>0$ (all four are independent of $R_{0}$ ) such that the following hold:

(1.4) $c_{\mu}^{-1} r^{d} \leqslant \mu(B(x, r)) \leqslant c_{\mu} r^{d}$

$$
\begin{array}{rr}
0<\mu_{x} \leqslant c_{\mu}, & \forall x \in V, \\
\inf _{x \in B(0, R)} \mu_{x} \geqslant R^{-\kappa}, & \forall R \geqslant R_{0},
\end{array}
$$

In particular, $(G, \mu)$ only satisfies the $d$-set condition uniformly for large scale under (1.4). For $p \geqslant 1$, let $L^{p}(V ; \mu)=\left\{f \in \mathbb{R}^{V}: \sum_{x \in V}|f(x)|^{p} \mu_{x}<\infty\right\}$, and $\|f\|_{p}$ be the $L^{p}(V ; \mu)$ norm of $f$ with respect to $\mu$. Let $L^{\infty}(V ; \mu)$ be the space of bounded measurable functions on $V$, and $\|f\|_{\infty}$ be the $L^{\infty}(V ; \mu)$ norm of $f$.

Suppose that $\left\{w_{x, y}: x, y \in V\right\}$ is a sequence such that $w_{x, y} \geqslant 0$ and $w_{x, y}=w_{y, x}$ for all $x \neq y$, and

$$
\sum_{y \in V: y \neq x} \frac{w_{x, y} \mu_{y}}{\rho(x, y)^{d+\alpha}}<\infty, \quad x \in V
$$

where $\alpha \in(0,2)$. For simplicity, we set $w_{x, x}=0$ for all $x \in V$. We can define a regular Dirichlet form $(D, \mathscr{F})$ as follows (see the first statement in [30, Theorem 3.2])

$$
\begin{aligned}
D(f, f) & =\frac{1}{2} \sum_{x, y \in V}(f(x)-f(y))^{2} \frac{w_{x, y}}{\rho(x, y)^{d+\alpha}} \mu_{x} \mu_{y} \\
\mathscr{F} & =\left\{f \in L^{2}(V ; \mu): D(f, f)<\infty\right\} .
\end{aligned}
$$


It is easy to verify that the infinitesimal generator $\mathcal{L}$ associated with $(D, \mathscr{F})$ is given by

$$
\mathcal{L} f(x)=\sum_{z \in V}(f(z)-f(x)) \frac{w_{x, z} \mu_{z}}{\rho(x, z)^{d+\alpha}} .
$$

Let $X:=\left(X_{t}\right)_{t \geqslant 0}$ be the symmetric Hunt process associated with $(D, \mathscr{F})$. When $\mu$ is a counting measure on $G$ (resp. $\mu_{x}$ is chosen to be satisfied that $1=\sum_{z \in V} \frac{w_{x, z} \mu_{z}}{\rho(x, z)^{d+\alpha}}$ for all $x \in V$ ), the associated process $X$ is called the variable speed random walk (resp. the constant speed random walk) in the literature.

For any subset $D \subset V$, let $\tau_{D}:=\inf \left\{t>0: X_{t} \notin D\right\}$ be the first exit time from $D$ for the process $X$. Denote by $X^{D}:=\left(X_{t}^{D}\right)_{t \geqslant 0}$ the Dirichlet process, i.e.,

$$
X_{t}^{D}:=\left\{\begin{array}{cc}
X_{t}, & \text { if } t<\tau_{D}, \\
\partial, & \text { if } t \geqslant \tau_{D},
\end{array}\right.
$$

where $\partial$ denotes the cemetery point. Let $p^{D}(t, x, y)$ be the Dirichlet heat kernel associated with the process $X^{D}$.

In the accompanied paper [24], we discussed the quenched invariance principle for conductance models with stable-like jumps. As a continuation of [24], we consider heat kernel estimates for the conductance models. The main difficulty here is due to that neither that conductances are uniformly elliptic (possibly degenerate) nor the global $d$-set condition is supposed to be satisfied. To illustrate our contribution, we state the following result for random conductance models on $\mathbb{L}:=\mathbb{Z}_{+}^{d_{1}} \times \mathbb{Z}^{d_{2}}$ with $d_{1}, d_{2} \in \mathbb{Z}_{+}:=\{0,1,2, \cdots\}$ such that $d_{1}+d_{2} \geqslant 1$ (i.e., $V=\mathbb{L}$ and the coefficients $w_{x, y}$ given in (1.5) are random variables).

Theorem 1.1. (Heat kernel estimates for Variable speed random walks) Let $V=\mathbb{L}$ with $d>4-2 \alpha$, and $\left\{w_{x, y}(\omega): x, y \in \mathbb{L}\right\}$ be a sequence of independent random variables on some probability space $\left(\Omega, \mathscr{F}_{\Omega}, \mathbb{P}\right)$ such that for any $x \neq y, w_{x, y}=w_{y, x} \geqslant 0$ and

$$
\sup _{x, y \in \mathbb{L}: x \neq y} \mathbb{P}\left(w_{x, y}=0\right)<2^{-4}, \quad \sup _{x, y \in \mathbb{L}: x \neq y}\left(\mathbb{E}\left[w_{x, y}^{p}\right]+\mathbb{E}\left[w_{x, y}^{-q} \mathbb{1}_{\left\{w_{x, y}>0\right\}}\right]\right)<\infty
$$

for some $p, q \in \mathbb{Z}_{+}$with

$$
p>\max \left\{\left(d+1+\theta_{0}\right) /\left(d \theta_{0}\right),(d+1) /\left(2 \theta_{0}(2-\alpha)\right)\right\}, \quad q>\left(d+1+\theta_{0}\right) /\left(d \theta_{0}\right),
$$

where $\theta_{0}:=\alpha /(2 d+\alpha)$. Let $\left(X_{t}^{\omega}\right)_{t \geqslant 0}$ be the symmetric Hunt process corresponding to the Dirichlet form $(D, \mathscr{F})$ above with random variables $\left\{w_{x, y}(\omega): x \neq y \in \mathbb{L}\right\}$ and $\mu$ being the counting measure on $\mathbb{L}$. Denote by $p^{\omega}(t, x, y)$ the heat kernel of the process $\left(X_{t}^{\omega}\right)_{t \geqslant 0}$. Then, $\mathbb{P}$-a.s. $\omega \in \Omega$, for any $x \in \mathbb{L}$, there is a constant $R_{x}(\omega) \geqslant 1$ such that for all $R>R_{x}(\omega)$ and for all $t>0$ and $y \in \mathbb{L}$ with $t \geqslant\left(|x-y| \vee R_{x}(\omega)\right)^{\theta \alpha}$,

$$
C_{1}\left(t^{-d / \alpha} \wedge \frac{t}{|x-y|^{d+\alpha}}\right) \leqslant p^{\omega}(t, x, y) \leqslant C_{2}\left(t^{-d / \alpha} \wedge \frac{t}{|x-y|^{d+\alpha}}\right),
$$

where $\theta \in(0,1)$ and $C_{1}, C_{2}>0$ are constants independent of $R_{x}(\omega), t, x$ and $y$.

Note that (1.7) is the typical heat kernel estimates for stable-like jumps, and it corresponds to the Gaussian estimates (1.1) for the nearest neighbor cases.

Let us explain some related work. As we mentioned above, there are only a few results for conductance models with stable-like (long range) jumps. When the conductances are uniformly elliptic and the global volume doubling condition holds, heat kernel estimates like (1.7) have been discussed, for instance in [9, 15, 47, 48]. In these aforementioned papers, a lot of arguments are heavily based on uniformly elliptic conductances, and two-sided pointwise bounds of conductances are also necessary and frequently used. The corresponding results for non-local Dirichlet forms on general metric measure spaces 
now have been obtained, and, in particular, the De Giorgi-Nash-Moser theory are developed, see [25, 26, 27, 28, 29, 42, 43] and the references therein. Crawford and Sly [32] proved on-diagonal heat kernel upper bounds for random walks on the infinite cluster of supercritical long range percolation, see [33] for the scaling limit of random walks on long range percolation clusters. Due to the singularity of long range percolation cluster, offdiagonal heat kernel estimates are still unknown and seem to be quite different from those for conductance models with stable-like jumps. As mentioned before, it does not seem that heat kernel estimates for conductance models with non-uniformly elliptic stable-like jumps and under non-uniformly volume doubling condition are available till now. In this paper we will address this problem completely.

We summarize some difficulties of our problem as follows.

(i) As for nearest neighbor non-uniformly elliptic conductance models, a usual (and powerful) idea is to establish first elliptic and parabolic Harnack inequalities, and then deduce heat kernel bounds. For example, see [2, 3, 4] for the recent study on ergodic environments of nearest neighbor random conductance models under some integrability conditions. However, we will prove that in the present setting elliptic Harnack inequalities do not hold even for large balls and so parabolic Harnack inequalities do not hold in general either, when conductances are not uniformly elliptic. This is totally different from uniformly elliptic stable-like jumps, see e.g. $[9,15,47,48]$, or uniformly elliptic stable-like jumps with variable orders on the Euclidean space $\mathbb{R}^{d}$, see e.g. [14]. We refer readers to Proposition 4.7 and Example 4.8 below for details.

(ii) In case of nearest neighbor non-uniformly elliptic conductance models, off-diagonal upper bounds of the heat kernel can be deduced from on diagonal upper bounds using the maximum principle initiated by Grigor'yan on manifolds [41] and developed in [40] on graphs, see e.g. the proofs of [22, Proposition 1.2] or [10, Proposition 3.3]. Because of the effect of long range jumps, such approach does not seem to be applicable in our model.

(iii) As mentioned before, in order to establish heat kernel estimates for uniformly elliptic stable-like jumps, pointwise upper and lower bounds of conductances are crucially used in $[9,15,47,48]$. In particular, uniform lower bounds of conductances yield Nash/Sobolev inequalities for the associated Dirichlet form, which in turn imply on-diagonal heat kernel upper bounds immediately. Furthermore, based on Nash/Sobolev inequalities, the Davies method was adopted in $[9,15,47,48]$ to derive off-diagonal upper bound estimates for heat kernel. However, in the setting of our paper Nash/Sobolev inequalities do not hold, and so the approaches above are not applicable.

To establish two-sided heat kernel estimates for long range and non-uniformly elliptic conductance models with stable-like jumps, we will apply the localization argument for Dirichlet heat kernel estimates, and then pass through these to global heat kernel estimates via the Dynkin-Hunt formula. For this, we make full use of estimates for the exit time of the process obtained in [24]. Though part of ideas in the proofs are motivated by the study of global heat kernel estimates for uniformly elliptic conductance models with stablelike jumps (for instance, see $[9,12]$ ), it seems that this is the first time to adopt them to investigate the corresponding Dirichlet heat kernel estimates for large time scale. In the proof, a lot of non-trivial modifications and new ideas are required. Actually, in this paper we will establish heat kernel estimates under a quite general framework beyond Theorem 1.1, see Theorem 2.8 and Theorem 2.12 below. In particular, only the $d$-set condition with large scale $(d$-Vol) and locally summable conditions on conductances (see Assumptions (HK1)-(HK3) below) are assumed. These conditions can be regarded as a generalization of "good ball" conditions for nearest neighbor conductance models in [10] into long range 
conductance models. As an application of a series of (large scale) probability estimates for exit times and regularity of parabolic harmonic functions as well as heat kernel estimates for large time, we can also justify the local limit theorem for our model, see Theorem 4.4 below.

The organization of this paper is as follows. The next section is devoted to heat kernel estimates for large time. This part is split into three subsections. We first consider ondiagonal upper bounds, later study off-diagonal upper bounds, and then lower bound estimates. In Section 3, we present some estimates for Green functions, and also give a consequence of elliptic Harnack inequalities. In the last section, we apply our previous results to random conductances with stable-like jumps.

\section{Heat Kernel Estimates: Large Time}

To obtain heat kernel estimates for large time, we need the following three assumptions on $\left\{w_{x, y}: x, y \in V\right\}$. We fix $0 \in V$, and define $B_{z}^{w}(x, r):=\left\{y \in B(x, r): w_{y, z}>0\right\}$ for all $x, z \in V$ and $r>0$. Set $B^{w}(x, r):=B_{x}^{w}(x, r)$ for simplicity.

Assumption (HK1). Suppose that there exist $R_{0} \geqslant 1, \theta \in(0,1), c_{0}>1 / 2$ and $C_{1}>0$ (all three are independent of $R_{0}$ ) such that

(i) For every $R>R_{0}$ and $R^{\theta} / 2 \leqslant r \leqslant 2 R$,

$$
\begin{gathered}
\sup _{x \in B(0,6 R)} \sum_{y \in V: \rho(x, y) \leqslant r} \frac{w_{x, y} \mu_{y}}{\rho(x, y)^{d+\alpha-2}} \leqslant C_{1} r^{2-\alpha}, \\
\mu\left(B_{z}^{w}(x, r)\right) \geqslant c_{0} \mu(B(x, r)), \quad x, z \in B(0,6 R),
\end{gathered}
$$

and

$$
\sup _{x \in B(0,6 R)} \sum_{y \in B^{w}\left(x, c_{*} r\right)} w_{x, y}^{-1} \mu_{y} \leqslant C_{1} r^{d}
$$

where $c_{*}:=8 c_{\mu}^{2 / d}$

(ii) For every $R>R_{0}$ and $r \geqslant R^{\theta} / 2$,

$$
\sup _{x \in B(0,6 R)} \sum_{y \in V: \rho(x, y)>r} \frac{w_{x, y} \mu_{y}}{\rho(x, y)^{d+\alpha}} \leqslant C_{1} r^{-\alpha} .
$$

Assumption (HK2). Suppose that for some fixed $\theta \in(0,1)$, there exist $R_{0} \geqslant 1$ and $C_{2}>0$ (independent of $R_{0}$ ) such that for every $R>R_{0}$ and $R^{\theta} / 2 \leqslant r \leqslant 2 R$,

$$
\sup _{x, y \in B(0,6 R)} \sum_{z \in V: \rho(z, y) \leqslant r} w_{x, z} \mu_{z} \leqslant C_{2} r^{d} .
$$

Assumption (HK3). Suppose that for some fixed $\theta \in(0,1)$, there exist $R_{0} \geqslant 1$ and $C_{3}>0$ (independent of $R_{0}$ ) such that for every $R>R_{0}$ and $R^{\theta} / 2 \leqslant r \leqslant 2 R$,

$$
\inf _{x, y \in B(0,6 R)} \sum_{z \in V: \rho(z, y) \leqslant r} w_{x, z} \mu_{z} \geqslant C_{3} r^{d} .
$$

Assumption (HK1) is a slight modification of [24, Assumption (Exi.)], which was used to derive the distribution and the expectation of exit time, see [24, Theorem 3.4] or Theorem 2.2 below. Actually, in [24] Assumption (HK1) is also adopted to yield the (large scale) Hölder regularity of associated parabolic functions, see [24, Theorem 3.8] or Theorem 2.10 in Subsection 2.3. We further note that when $\alpha \in(0,1)$, one can replace Assumption (HK1) by [24, Assumption (Exi'.)] to deduce the distribution and the expectation of exit time, which may refine some conditions in Assumption (HK1). The details are left to interested readers. 


\subsection{Upper bounds of the heat kernel.}

\subsubsection{On diagonal upper bounds of the heat kernel.}

Proposition 2.1. (On diagonal upper bound for the Dirichlet heat kernel) Assume that Assumption ( $d$ - Vol) holds with some $\theta \in(0,1), \kappa>0$ and $R_{0} \geqslant 1$, and that there exists $R_{0}^{\prime} \geqslant 1$ such that for all $R \geqslant R_{0}^{\prime}$ and $R^{\theta} \leqslant r \leqslant R$,

$$
\mu\left(B_{z}^{w}(x, r)\right) \geqslant c_{0} \mu(B(x, r)), \quad x, z \in B(0,2 R)
$$

and

$$
\sup _{x \in B(0,2 R)} \sum_{y \in B^{w}(x, 2 r)} w_{x, y}^{-1} \mu_{y} \leqslant C_{0} r^{d},
$$

where $c_{0}>1 / 2$ and $C_{0}>0$ are independent of $R_{0}, R_{0}^{\prime}, R$ and $r$. Then, for every $\theta^{\prime} \in(\theta, 1)$, there exists a constant $R_{1} \geqslant 1$ such that for all $R>R_{1}, x_{1}, x_{2} \in B(0, R)$ and $t \geqslant R^{\theta^{\prime} \alpha}$,

$$
p^{B(0, R)}\left(t, x_{1}, x_{2}\right) \leqslant C_{1} t^{-d / \alpha},
$$

where $C_{1}$ is a positive constant independent of $R_{0}, R_{0}^{\prime}, R_{1}, R, x_{1}, x_{2}$ and $t$.

Proof. The proof is to some extent similar to that of [24, Proposition 2.2], which is concerned with on diagonal upper bounds for global heat kernel of truncated processes. We will provide the complete proof here for convenience of readers. Noticing that, by the Cauchy-Schwarz inequality, $p\left(2 t, x_{1}, x_{2}\right) \leqslant p\left(t, x_{1}, x_{1}\right)^{1 / 2} p\left(t, x_{2}, x_{2}\right)^{1 / 2}$ for any $x_{1}, x_{2} \in V$ and $t>0$, it suffices to show (2.3) for the case $x_{1}=x_{2}$. We will split the proof into three steps.

Step (1) We first prove that there are constants $R_{2} \geqslant 1$ and $C_{2}>0$ (independent of $R_{2}$ ) such that for any $R>R_{2}, x \in B(0, R), R^{\theta} \leqslant r<R$ and any measurable function $f$ on $V$,

$$
\sum_{z \in B(x, r)}\left(f(z)-(f)_{B^{w}(z, r)}\right)^{2} \mu_{z} \leqslant C_{2} r^{\alpha} \sum_{z \in B(x, r), y \in B(x, 2 r)}(f(z)-f(y))^{2} \frac{w_{z, y}}{\rho(z, y)^{d+\alpha}} \mu_{y} \mu_{z},
$$

where for $A \subset V$,

$$
(f)_{A}:=\frac{1}{\mu(A)} \sum_{z \in A} f(z) \mu_{z} .
$$

Indeed, for every $R>R_{2}:=R_{0}^{1 / \theta} \vee R_{0}^{\prime}$ with $R_{0}$ and $R_{0}^{\prime}$ being the constants in Assumption $\left(d\right.$-Vol) and Proposition 2.1 respectively, $x \in B(0, R)$ and $R^{\theta} \leqslant r \leqslant R$, we have

$$
\begin{aligned}
& \sum_{z \in B(x, r)}\left(f(z)-(f)_{B^{w}(z, r)}\right)^{2} \mu_{z} \\
= & \sum_{z \in B(x, r)}\left(\frac{1}{\mu\left(B^{w}(z, r)\right)} \sum_{y \in B^{w}(z, r)}(f(z)-f(y)) \mu_{y}\right)^{2} \mu_{z} \\
\leqslant & \frac{c_{1}}{r^{2 d}} \sum_{z \in B(x, r)}\left[\left(\sum_{y \in B^{w}(z, r)}(f(z)-f(y))^{2} \frac{w_{z, y} \mu_{y}}{\rho(z, y)^{d+\alpha}}\right)\left(\sum_{y \in B^{w}(z, r)} w_{z, y}^{-1} \rho(z, y)^{d+\alpha} \mu_{y}\right)\right] \mu_{z} \\
\leqslant & c_{2} r^{-d+\alpha}\left(\sup _{z \in B(0,2 R)} \sum_{y \in B^{w}(z, 2 r)} w_{z, y}^{-1} \mu_{y}\right)\left(\sum_{z \in B(x, r), y \in B(x, 2 r)}(f(z)-f(y))^{2} \frac{w_{z, y} \mu_{y} \mu_{z}}{\rho(z, y)^{d+\alpha}}\right) \\
\leqslant & \left.c_{3} r^{\alpha}\right) \\
& \sum_{z \in B(x, r), y \in B(x, 2 r)}(f(z)-f(y))^{2} \frac{w_{z, y}}{\rho(z, y)^{d+\alpha}} \mu_{y} \mu_{z},
\end{aligned}
$$

where in the first inequality we used (1.4), (2.1) and the Cauchy-Schwarz inequality, and the third inequality is due to (2.2). 
Step (2) For any $x \in B(0, R)$ and $R>R_{2}$, let $f_{t}(z)=p^{B(0, R)}(t, x, z)$ and $\psi(t)=$ $p^{B(0, R)}(2 t, x, x)$ for all $z \in V$ and $t \geqslant 0$. Then, $\psi(t)=\sum_{z \in B(0, R)} f_{t}(z)^{2} \mu_{z}$ and

$$
\psi^{\prime}(t)=2 \sum_{z \in B(0, R)} \frac{d f_{t}(z)}{d t} f_{t}(z) \mu_{z}=-\sum_{z, y \in V}\left(f_{t}(z)-f_{t}(y)\right)^{2} \frac{w_{z, y}}{\rho(z, y)^{d+\alpha}} \mu_{y} \mu_{z} .
$$

In particular, the second equality above, yielded by the integration by parts formula, holds since the finite summation over variable $z$ together with (1.5) ensures the integrability of the associated terms.

Let $2 R^{\theta} \leqslant r(t) \leqslant R$ be a constant to be determined later. Let $B\left(x_{i}, r(t) / 2\right)(i=$ $1, \cdots, m)$ be the maximal collection of disjoint balls with centers in $B(0, R)$. Set $B_{i}=$ $B\left(x_{i}, r(t)\right)$ and $B_{i}^{*}=B\left(x_{i}, 2 r(t)\right)$ for $1 \leqslant i \leqslant m$. Note that $B(0, R) \subset \bigcup_{i=1}^{m} B_{i} \subset$ $B(0, R+r(t))$; moreover, if $x \in B(0, R+r(t)) \cap B_{i}^{*}$ for some $1 \leqslant i \leqslant m$, then $B\left(x_{i}, r(t) / 2\right) \subset$ $B(x, 3 r(t))$. So

$$
c_{4} r(t)^{d} \geqslant \mu(B(0,3 r(t))) \geqslant \sum_{i=1}^{m} \mathbb{1}_{\left\{x \in B_{i}^{*}\right\}} \mu\left(B\left(x_{i}, r(t) / 2\right)\right) \geqslant c_{5} r(t)^{d} \sharp\left\{i: x \in B_{i}^{*}\right\},
$$

where $\sharp A$ is a number of elements in the set $A$ for any $A \subset \mathbb{Z}$, and we used (1.4) and the fact that $r(t) \geqslant 2 r_{0}$. Thus, any $x \in B(0, R+r(t))$ is in at most $c_{6}:=c_{4} / c_{5}$ of the ball $B_{i}^{*}$ (hence at most $c_{6}$ of the ball $B_{i}$ ), and

$$
\sum_{i=1}^{m} \sum_{z \in B_{i}}=\sum_{i=1}^{m} \sum_{z \in B(0, R+r(t))} \mathbb{1}_{B_{i}}(z)=\sum_{z \in B(0, R+r(t))} \sum_{i=1}^{m} \mathbb{1}_{B_{i}}(z) \leqslant c_{6} \sum_{z \in B(0, R+r(t))} .
$$

Noting that $R>R_{2}$ and $2 R^{\theta} \leqslant r(t) \leqslant R$, we obtain

$$
\begin{aligned}
& \sum_{z, y \in V}\left(f_{t}(z)-f_{t}(y)\right)^{2} \frac{w_{z, y}}{\rho(z, y)^{d+\alpha}} \mu_{y} \mu_{z} \\
& \geqslant \frac{1}{c_{6}} \sum_{i=1}^{m} \sum_{z \in B_{i}} \sum_{y \in B_{i}^{*}}\left(f_{t}(z)-f_{t}(y)\right)^{2} \frac{w_{z, y}}{\rho(z, y)^{d+\alpha}} \mu_{y} \mu_{z} \\
& \geqslant \frac{c_{7}}{r(t)^{\alpha}}\left[\sum_{i=1}^{m} \sum_{z \in B_{i}} f_{t}^{2}(z) \mu_{z}-2 \sum_{i=1}^{m} \sum_{z \in B_{i}} f_{t}(z)\left(f_{t}\right)_{B^{w}(z, r(t))} \mu_{z}\right]=: \frac{c_{7}}{r(t)^{\alpha}}\left(I_{1}-I_{2}\right),
\end{aligned}
$$

where the first inequality is due to (2.5) and in the second inequality we used (2.4).

Furthermore, we have

$$
I_{1} \geqslant \sum_{z \in \cup_{i=1}^{m} B_{i}} f_{t}^{2}(z) \mu_{z} \geqslant \sum_{z \in B(0, R)} f_{t}^{2}(z) \mu_{z}=\psi(t) .
$$

Note that $2 R^{\theta} \leqslant r(t) \leqslant R$. According to (1.4), (2.1) and the fact that $\sum_{z \in V} f_{t}(z) \mu_{z} \leqslant 1$, we have

$$
\sup _{z \in B(0,2 R)}\left(f_{t}\right)_{B^{w}(z, r(t))} \leqslant \sup _{z \in B(0,2 R)} \mu\left(B^{w}(z, r(t))\right)^{-1} \cdot \sum_{z \in V} f_{t}(z) \mu_{z} \leqslant c_{7}^{*} r(t)^{-d} .
$$

Hence, by (2.5),

$$
I_{2} \leqslant c_{7}^{*} r(t)^{-d} \sum_{i=1}^{m} \sum_{z \in B_{i}} f_{t}(z) \mu_{z} \leqslant c_{5} c_{7}^{*} r(t)^{-d} \sum_{z \in B(0, R+r(t))} f_{t}(z) \mu_{z} \leqslant c_{5} c_{7}^{*} r(t)^{-d} .
$$

Therefore, combining with all the estimates above, we obtain that for every $2 R^{\theta} \leqslant r(t) \leqslant R$ with $R>R_{2}$,

$$
\psi^{\prime}(t) \leqslant-c_{8} r(t)^{-\alpha}\left(\psi(t)-c_{9} r(t)^{-d}\right) .
$$


Step (3) For any $\theta^{\prime} \in(\theta, 1)$ and any $R>R_{2}$ large enough, we claim that there exists $t_{0} \in\left[R^{\theta \alpha}, R^{\theta^{\prime} \alpha}\right]$ such that

$$
\left(\frac{1}{2 c_{9}} \psi\left(t_{0}\right)\right)^{-1 / d} \geqslant 2 R^{\theta}
$$

Indeed, assume that (2.7) does not hold. Then, for all $R^{\theta \alpha} \leqslant t \leqslant R^{\theta^{\prime} \alpha}$,

$$
\left(\frac{1}{2 c_{9}} \psi(t)\right)^{-1 / d}<2 R^{\theta}
$$

which implies that $\psi(t) \geqslant 2 c_{9}\left(2 R^{\theta}\right)^{-d}$ for all $R^{\theta \alpha} \leqslant t \leqslant R^{\theta^{\prime} \alpha}$. Therefore, taking $r(t)=2 R^{\theta}$ in (2.6), we find that for all $R^{\theta \alpha} \leqslant t \leqslant R^{\theta^{\prime} \alpha}$,

$$
\psi^{\prime}(t) \leqslant-\frac{c_{8}}{2}\left(2 R^{\theta}\right)^{-\alpha} \psi(t)
$$

which along with the fact $\psi(t) \leqslant \mu_{x}^{-1} \leqslant R^{\kappa}$ for all $t>0$ and $x \in B(0, R)$ (due to (1.3)) yields that for all $R^{\theta \alpha} \leqslant t \leqslant R^{\theta^{\prime} \alpha}$,

$$
\psi(t) \leqslant R^{\kappa} e^{-\frac{c_{8}}{2}\left(2 R^{\theta}\right)^{-\alpha}\left(t-R^{\theta \alpha}\right)} .
$$

In particular,

$$
\psi\left(R^{\theta^{\prime} \alpha}\right) \leqslant R^{\kappa} e^{-\frac{c_{8}}{2}\left(2 R^{\theta}\right)^{-\alpha}\left(R^{\theta^{\prime} \alpha}-R^{\theta \alpha}\right)} .
$$

On the other hand, by (2.8), we have $\psi\left(R^{\theta^{\prime} \alpha}\right) \geqslant 2 c_{9}\left(2 R^{\theta}\right)^{-d}$. Thus, there is a contradiction between these two inequalities above for $R$ large enough. In particular, there exists $R_{1}>R_{2}$ such that (2.7) holds for all $R>R_{1}$.

From now on, we may and do assume that (2.7) holds for all $R>R_{1}$. Since $t \mapsto \psi(t)$ is non-increasing on $(0, \infty)$ and $t_{0} \leqslant R^{\theta^{\prime} \alpha}$, we have that for all $R^{\theta^{\prime} \alpha} \leqslant t \leqslant R^{\alpha}$,

$$
\left(\frac{1}{2 c_{9}} \psi(t)\right)^{-1 / d} \geqslant 2 R^{\theta} \text {. }
$$

Let

$$
\tilde{t}_{0}:=\sup \left\{t>0:\left(\frac{1}{2 c_{9}} \psi(t)\right)^{-1 / d}<R / 2\right\} .
$$

By the non-increasing property of $\psi$ on $(0, \infty)$ again, if $\tilde{t}_{0} \leqslant 2 R^{\theta^{\prime} \alpha}$, then for $2 R^{\theta^{\prime} \alpha} \leqslant t \leqslant R^{\alpha}$

$$
\psi(t) \leqslant \psi\left(\tilde{t}_{0}\right)=2 c_{9}(R / 2)^{-d} \leqslant c_{10} t^{-d / \alpha} .
$$

If $\tilde{t}_{0}>2 R^{\theta^{\prime} \alpha}$, then

$$
2 R^{\theta} \leqslant\left(\frac{1}{2 c_{9}} \psi(t)\right)^{-1 / d} \leqslant R / 2
$$

for all $R^{\theta^{\prime} \alpha} \leqslant t \leqslant \tilde{t}_{0}$. Taking $r(t)=\left(\frac{1}{2 c_{9}} \psi(t)\right)^{-1 / d}$ in $(2.6)$, we know that for all $R^{\theta^{\prime} \alpha} \leqslant$ $t \leqslant \tilde{t}_{0}, \psi^{\prime}(t) \leqslant-c_{11} \psi(t)^{1+\alpha / d}$. Hence, for all $2 R^{\theta^{\prime} \alpha} \leqslant s \leqslant \tilde{t}_{0}$,

$$
\psi(s) \leqslant c_{12}\left(s-R^{\theta^{\prime} \alpha}+\psi\left(R^{\theta^{\prime} \alpha}\right)^{-\alpha / d}\right)^{-d / \alpha} \leqslant c_{13} s^{-d / \alpha} .
$$

If $2 R^{\theta^{\prime} \alpha} \leqslant \tilde{t}_{0} \leqslant R^{\alpha}$, then for all $\tilde{t}_{0} \leqslant s \leqslant R^{\alpha}$, we have

$$
\psi(s) \leqslant \psi\left(\tilde{t}_{0}\right)=2 c_{9}(R / 2)^{-d} \leqslant c_{15} s^{-d / \alpha} .
$$

If $\tilde{t}_{0}>R^{\alpha}$, then (2.3) holds similarly for every $2 R^{\theta^{\prime} \alpha} \leqslant t \leqslant R^{\alpha}$. Combining all the estimates above and choosing $\theta^{\prime}$ larger if necessary, we can obtain (2.3) for all $R>R_{1}$ and $2 R^{\theta^{\prime} \alpha} \leqslant t \leqslant R^{\alpha}$.

Finally, for every $x \in B(0, R)$ and $t>R^{\alpha}$, taking $N \geqslant R$ such that $x \in B(0, N)$ and $2 N^{\theta^{\prime} \alpha} \leqslant t \leqslant N^{\alpha}$, we can get

$$
p^{B(0, R)}(t, x, x) \leqslant p^{B(0, N)}(t, x, x) \leqslant C_{1} t^{-d / \alpha},
$$


which implies (2.3) also holds for all $t>R^{\alpha}$. Altogether, we obtain (2.3) for $t \geqslant 2 R^{\theta^{\prime} \alpha}$ for all $R>R_{1}$. By changing the choice of $R_{1}$ (namely taking $2^{1 /\left(\theta^{\prime} \alpha\right)} R_{1}$ as a new $R_{1}$ ), we have (2.3) for $t \geqslant R^{\theta^{\prime} \alpha}$ for all $R>R_{1}$, and the proof is complete.

The following statement is an improvement of [24, Theorem 3.4], which was proven under the global $d$-set condition.

Theorem 2.2. Suppose that Assumptions $(d-\mathrm{Vol})$ and $(\mathrm{HK} 1)$ hold with some constants $\theta \in(0,1)$ and $R_{0} \geqslant 1$. Then, for every $\theta^{\prime} \in(\theta, 1)$, there exist constants $\delta \in(\theta, 1), R_{1} \geqslant 1$ such that the following hold for all $R>R_{1}$ and $R^{\delta} \leqslant r \leqslant R$,

$$
\begin{gathered}
\sup _{x \in B(0,2 R)} \mathbb{P}_{x}\left(\tau_{B(x, r)} \leqslant C_{0} r^{\alpha}\right) \leqslant 1 / 4, \\
\sup _{x \in B(0,2 R)} \mathbb{P}_{x}\left(\tau_{B(x, r)} \leqslant t\right) \leqslant C_{1}\left(\frac{t}{r^{\alpha}}\right)^{1 / 2}\left[1 \vee \log \left(\frac{r^{\alpha}}{t}\right)\right], \quad t \geqslant r^{\theta^{\prime} \alpha}, \\
C_{2} r^{\alpha} \leqslant \inf _{x \in B(0,2 R)} \mathbb{E}_{x}\left[\tau_{B(x, r)}\right] \leqslant \sup _{x \in B(0,2 R)} \mathbb{E}_{x}\left[\tau_{B(x, r)}\right] \leqslant C_{1} r^{\alpha},
\end{gathered}
$$

where $C_{0}, C_{1}$ and $C_{2}>0$ are independent of $R_{0}, R_{1}, R, r$ and $t$.

Proof. The proof is heavily motivated by that of [24, Theorem 3.4], and we only present main different points here.

First, it is seen from the proof of [24, Theorem 3.4] that the crucial point for the required assertions is to verify moment estimates (see [24, Proposition 2.3]) for the truncation of localized processes under Assumptions ( $d$-Vol) and (HK1). A difference from [24, Section 2.2 ] is, here we will adopt the localization approach by using reflected Dirichlet forms on bounded sets. In details, we consider the following localization of truncated reflected Dirichlet form on the ball $B(0,6 R)$ :

$$
\begin{aligned}
\hat{D}^{R, R}(f, f) & =\sum_{x, y \in B(0,6 R): \rho(x, y) \leqslant R}(f(x)-f(y))^{2} \frac{w_{x, y}}{\rho(x, y)^{d+\alpha}} \mu_{x} \mu_{y}, \quad f \in \hat{\mathscr{F}} R, R, \\
\hat{\mathscr{F}} R, R & =\left\{f \in L^{2}(B(0,6 R) ; \mu): \hat{D}^{R, R}(f, f)<\infty\right\} .
\end{aligned}
$$

Let $\left(\hat{X}_{t}^{R, R}\right)_{t \geqslant 0}$ be the Hunt process associated with $\left(\hat{D}^{R, R}, \hat{\mathscr{F}} R, R\right)$. Regard $B(0,6 R)$ as the whole space $V$ in [24, Section 2.2]. By carefully tracking the proofs of [24, Proposition 2.2 and Proposition 2.3] and noticing that the lower bound of $\mu_{x}$ was not used in the proofs, we can prove that, under Assumptions ( $d$-Vol) and (HK1), for every $\theta^{\prime} \in(\theta, 1)$, there exist $R_{1} \geqslant 1$ and $C_{3}>0$ (independent of $\left.R_{1}\right)$ such that for all $x \in B(0,6 R)$,

$$
\mathbb{E}_{x}\left[\rho\left(\hat{X}_{t}^{R, R}, x\right)\right] \leqslant C_{3} R\left(\frac{t}{R^{\alpha}}\right)^{1 / 2}\left[1+\log \left(\frac{R^{\alpha}}{t}\right)\right], \quad R^{\theta^{\prime} \alpha} \leqslant t \leqslant R^{\alpha} .
$$

This along with the proof of [24, Proposition 3.2] further yields that for all $x_{0} \in B(0,2 R)$ and $t \geqslant R^{\theta^{\prime} \alpha}$

$$
\mathbb{P}_{x}\left(\hat{\tau}_{B\left(x_{0}, R\right)}^{R, R} \leqslant t\right) \leqslant C_{4}\left(\frac{t}{R^{\alpha}}\right)^{1 / 2}\left[1+\log \left(\frac{R^{\alpha}}{t}\right)\right]
$$

where $\hat{\tau}_{D}^{R, R}$ denotes the first exit time from $D \subset B(0,6 R)$ for the process $\left(\hat{X}_{t}^{R, R}\right)_{t \geqslant 0}$.

Next, we define the truncated Dirichlet form $\left(D^{R}, \mathscr{F}^{R}\right)$ as follows

$$
\begin{aligned}
D^{R}(f, f) & =\sum_{x, y \in V: \rho(x, y) \leqslant R}(f(x)-f(y))^{2} \frac{w_{x, y}}{\rho(x, y)^{d+\alpha}} \mu_{x} \mu_{y}, \quad f \in \mathscr{F}^{R}, \\
\mathscr{F}^{R} & =\left\{f \in L^{2}(V ; \mu): D^{R}(f, f)<\infty\right\} .
\end{aligned}
$$


Let $\left(X^{R}\right)_{t \geqslant 0}$ be the Hunt process associated with $\left(D^{R}, \mathscr{F}^{R}\right)$. Then, it is not difficult to verify that for any $x_{0} \in B(0,2 R)$ and $t>0$,

$$
\mathbb{P}_{x_{0}}\left(\tau_{B\left(x_{0}, R\right)}^{R} \leqslant t\right)=\mathbb{P}_{x_{0}}\left(\hat{\tau}_{B\left(x_{0}, R\right)}^{R, R} \leqslant t\right),
$$

where $\tau_{D}^{R}$ denotes the first exit time from $D \subset V$ for the process $\left(X_{t}^{R}\right)_{t \geqslant 0}$.

Therefore, putting (2.12), (2.13), [24, Lemma 3.1] and Assumption (HK1) (ii) together, we find that for all $x_{0} \in B(0,2 R)$ and $t>0$,

$$
\mathbb{P}_{x_{0}}\left(\tau_{B\left(x_{0}, R\right)} \leqslant t\right) \leqslant C_{5}\left(\frac{t}{R^{\alpha}}\right)^{1 / 2}\left[1+\log \left(\frac{R^{\alpha}}{t}\right)\right] .
$$

Hence, the desired assertion follows from this estimate and the argument of [24, Theorem 3.4].

We now prove the global on-diagonal upper bound.

Proposition 2.3. (On diagonal upper bound for the heat kernel) Suppose that Assumptions $(d-\mathbf{V o l})$ and $(\mathbf{H K} 1)$ hold with some constants $\theta \in(0, \alpha /(2 d+\alpha))$ and $R_{0} \geqslant$ 1. Then, for any $\theta^{\prime} \in(\theta, \alpha /(2 d+\alpha))$, there exists a constant $R_{1} \geqslant 1$ such that for all $x, y \in V$ and $t \geqslant\left(R_{1} \vee \rho(0, x) \vee \rho(0, y)\right)^{\theta^{\prime} \alpha}$,

$$
p(t, x, y) \leqslant C_{1}\left(1 \vee \mu_{y}^{-1}\right) t^{-d / \alpha},
$$

where $C_{1}>0$ is a constant independent of $R_{0}, R_{1}, x, y$ and $t$. In particular, for any $\theta^{\prime} \in(\theta, \alpha /(2 d+\alpha))$, there exists a constant $T_{0}>0$ such that for all $t>T_{0}$ and $x, y \in$ $B\left(0, t^{1 /\left(\theta^{\prime} \alpha\right)}\right)$, (2.14) holds with constant $C_{1}>0$ independent of $R_{0}, T_{0}, x, y$ and $t$.

Proof. According to the Dynkin-Hunt formula, for every $N \geqslant 2 R \geqslant 1, x, y \in B(0, R)$ and $t>0$

$$
\begin{aligned}
p(t, x, y) & =p^{B(0, N)}(t, x, y)+\mathbb{E}_{x}\left[p\left(t-\tau_{B(0, N)}, X_{\tau_{B(0, N)},}, y\right) \mathbb{1}_{\left\{t>\tau_{B(0, N)}\right\}}\right] \\
& =: J_{1, N}(t)+J_{2, N}(t) .
\end{aligned}
$$

According to (2.3) and (2.10), for any $\varepsilon \in(0,1 / 2)$ and $\theta^{\prime} \in(\theta, 1)$, we can find a constant $R_{1} \geqslant 1$ large enough such that for every $N>2 R_{1}, x \in B(0, R)$ and $t \geqslant N^{\theta^{\prime} \alpha}$,

$$
J_{1, N}(t) \leqslant c_{1} t^{-d / \alpha}
$$

and

$$
J_{2, N}(t) \leqslant \mu_{y}^{-1} \mathbb{P}_{x}\left(\tau_{B(0, N)}<t\right) \leqslant \mu_{y}^{-1} \mathbb{P}_{x}\left(\tau_{B(x, N / 2)}<t\right) \leqslant c_{1}\left(t N^{-\alpha}\right)^{(1 / 2)-\varepsilon} \mu_{y}^{-1},
$$

where in the inequality above we used the facts that $p(t, x, y) \leqslant \mu_{y}^{-1}$ for all $x, y \in V$, and $B(x, N / 2) \subset B(0, N)$ for any $x \in B(0, R)$ with $2 R \leqslant N$.

Let $N_{0}(t):=\left[t^{\left(2 d /\left(\alpha^{2}(1-2 \varepsilon)\right)\right)+(1 / \alpha)}\right]$. For any $\theta \in(0, \alpha /(2 d+\alpha))$ and $\theta^{\prime} \in(\theta, \alpha /(2 d+\alpha))$, we take $\varepsilon \in(0,1 / 2)$ such that

$$
\left(\frac{2 d}{\alpha^{2}(1-2 \varepsilon)}+\frac{1}{\alpha}\right) \theta^{\prime} \alpha=1 .
$$

Then, for all $R>R_{1}$ and $t \geqslant(3 R)^{\theta^{\prime} \alpha}$ with $R>R_{1}$, they hold that $t \geqslant N_{0}(t)^{\theta^{\prime} \alpha}$ and $N_{0}(t) \geqslant 2 R>2 R_{1}$. So, taking $N=N_{0}(t)$ in (2.15) and (2.16), we obtain that for all $R>R_{1}, t \geqslant(3 R)^{\theta^{\prime} \alpha}$ and $x, y \in B(0, R)$,

$$
\begin{aligned}
p(t, x, y) & \leqslant J_{1, N_{0}(t)}(t)+J_{2, N_{0}(t)}(t) \leqslant c_{1}\left(1 \vee \mu_{y}^{-1}\right)\left(t^{-d / \alpha}+\left(t N_{0}(t)^{-\alpha}\right)^{(1 / 2)-\varepsilon}\right) \\
& \leqslant c_{2}\left(1 \vee \mu_{y}^{-1}\right) t^{-d / \alpha} .
\end{aligned}
$$

Changing $\theta^{\prime}$ a little large if necessary, without loss of generality we may and can assume that the estimate above holds for all $t \geqslant R^{\theta^{\prime} \alpha}$. Therefore, (2.14) follows immediately by 
choosing $R=R_{1} \vee \rho(0, x) \vee \rho(0, y)$. Furthermore, choosing $T_{0}=R_{1}^{\theta^{\prime} \alpha}$ and $R=t^{1 /\left(\theta^{\prime} \alpha\right)}$ respectively in the conclusion above, we can get the second assertion.

2.2. Off diagonal upper bound estimates. We first recall the following Lévy system formula, see e.g. [25, Lemma 4.7] or [26, Appendix A].

Lemma 2.4. For any $x \in V$, stopping time $\tau$ (with respect to the natural filtration of the precess $X)$, and non-negative measurable function $f$ on $[0, \infty) \times V \times V$ with $f(s, z, z)=0$ for all $z \in V$ and $s \geqslant 0$, we have

$$
\mathbb{E}_{x}\left[\sum_{s \leqslant \tau} f\left(s, X_{s-}, X_{s}\right)\right]=\mathbb{E}_{x}\left[\int_{0}^{\tau}\left(\sum_{z \in V} f\left(s, X_{s}, z\right) \frac{w_{X_{s}, z} \mu_{z}}{\rho\left(X_{s}, z\right)^{d+\alpha}}\right) d s\right] .
$$

Lemma 2.5. Suppose that Assumption (HK2) holds with constants $\theta \in(0,1)$ and $R_{0} \geqslant 1$. Then there exists a constant $R_{1} \geqslant 1$ such that for all $R>R_{1}, R^{\theta} \leqslant s \leqslant r / 2 \leqslant R$, $x_{0} \in B(0, R), x \in B\left(x_{0}, r / 2\right), y \in B\left(x_{0}, 2 r\right)^{c} \cap B(0, R)$ and $t>0$,

$$
\mathbb{P}_{x}\left(\tau_{B\left(x_{0}, r\right)} \leqslant t, X_{\tau_{B\left(x_{0}, r\right)}} \in B(y, s)\right) \leqslant \frac{C_{1} t s^{d}}{r^{d+\alpha}}
$$

where $C_{1}>0$ is independent of $x_{0}, x, R_{0}, R_{1}, R, s$ and $t$.

Proof. By Assumption (HK2), we know that there exists $R_{2} \geqslant 1$ such that for all $R>R_{2}$, $x, y \in B(0,2 R)$ and $R^{\theta} \leqslant s \leqslant R$,

$$
\sum_{z \in V: \rho(y, z) \leqslant s} w_{x, z} \mu_{z} \leqslant c_{1} s^{d}
$$

Then, according to (2.17), for any $x_{0} \in B(0, R), x \in B\left(x_{0}, r / 2\right)$ and $y \in B\left(x_{0}, 2 r\right)^{c} \cap$ $B(0, R), R^{\theta} \leqslant s \leqslant r / 2 \leqslant R$ and $t>0$,

$$
\begin{aligned}
\mathbb{P}_{x}\left(\tau_{B\left(x_{0}, r\right)} \leqslant t, X_{\tau_{B\left(x_{0}, r\right)}} \in B(y, s)\right) & \leqslant \mathbb{E}_{x}\left[\int_{0}^{t \wedge \tau_{B\left(x_{0}, r\right)}} \sum_{u \in V: \rho(u, y) \leqslant s} \frac{w_{X_{v}, u} \mu_{u}}{\rho\left(X_{v}, u\right)^{d+\alpha}} d v\right] \\
& \leqslant c_{2} t r^{-d-\alpha} \sup _{z, y \in B(0,2 R)} \sum_{u \in V: \rho(u, y) \leqslant s} w_{z, u} \mu_{u} \\
& \leqslant c_{3} t s^{d} r^{-d-\alpha}
\end{aligned}
$$

where in the second inequality we used the fact that

$$
\rho(u, v) \geqslant \rho(y, v)-\rho(y, u) \geqslant \rho\left(y, x_{0}\right)-\rho\left(x_{0}, v\right)-\rho(y, u) \geqslant r / 2
$$

for all $v \in B\left(x_{0}, r\right)$ and $u \in B(y, s)$, and the last inequality is due to (2.19).

Proposition 2.6. (Off diagonal upper bound for the Dirichlet heat kernel) Suppose that Assumptions ( $d$-Vol), (HK1) and (HK2) hold with $\theta \in(0,1)$ and $R_{0} \geqslant 1$. Then, for every $\theta^{\prime} \in(\theta, 1)$, there exists a constant $R_{1} \geqslant 1$ such that for all $R>R_{1}$, $x, y \in B(0, R)$ and $t \geqslant R^{\theta^{\prime} \alpha}$,

$$
p^{B(0, R)}(t, x, y) \leqslant C_{1}\left(t^{-d / \alpha} \wedge \frac{t}{\rho(x, y)^{d+\alpha}}\right),
$$

where $C_{1}>0$ is independent of $x, y, R_{0}, R_{1}, R$ and $t$.

Proof. We follow the proof of [12, Theorem $1.2(\mathrm{~b}) \Rightarrow(\mathrm{a})]$ with some required modifications due to the large scale setting. In the proof below the constant $c$ will be changed from line to line, and will be independent of $x, y, R_{0}, R_{1}, R$ and $t$.

For any $q \in[0, \infty)$ and $N \geqslant 1$, we call $\left(H_{q, N}\right)$ as follows: 
$\left(H_{q, N}\right)$ there is a constant $c>0$ such that for all $R \geqslant R_{1}$, and $x, y \in B(0, R)$,

$$
p^{B(0, R)}(t, x, y) \leqslant c t^{-d / \alpha}\left(\frac{t}{\rho(x, y)^{\alpha}}\right)^{q}, \quad t \geqslant N R^{\theta^{\prime} \alpha} .
$$

In particular, by $(2.3),\left(H_{0, N}\right)$ holds for all $N \geqslant 1$. Now, we will prove that

(i) If $\left(H_{q, N}\right)$ holds with some $0 \leqslant q<d / \alpha$, then $\left(H_{q+\delta, 2 N}\right)$ holds for every $\delta \in(0,1 / 2)$.

(ii) If $\left(H_{q, N}\right)$ holds with some $q>d / \alpha$, then $\left(H_{(1+(d / \alpha)) \wedge(q+\delta), 2 N}\right)$ holds for every $\delta \in(0,1 / 2)$.

Suppose that (i) and (ii) hold true. Since the iteration from $q=0$ and $q=1+(d / \alpha)$ only takes finite times, we can get $(2.20)$ by taking $\theta^{\prime}$ a little bit larger. In the following, we will prove (i) and (ii) respectively.

Step (1) We assume that $\left(H_{q, N}\right)$ holds with $0 \leqslant q<d / \alpha$. Let $t \geqslant 2 N R^{\theta^{\prime} \alpha}$, and $x, y \in$ $B(0, R)$. If $\rho(x, y) \leqslant 8 t^{1 / \alpha}$, then, by $\left(H_{0, N}\right),\left(H_{q+\delta, 2 N}\right)$ holds for every $\delta \in(0,1 / 2)$. Next, we suppose that $\rho(x, y)>8 t^{1 / \alpha}$. Set $\rho_{0}=t^{1 / \alpha}$ and $r=\rho(x, y) / 2$, so that $r>4 \rho_{0}$. Applying [12, Lemma 2.1] to the Dirichlet semigroup $\left(P_{t}^{B(0, R)}\right)_{t \geqslant 0}$ with $U=B(x, r) \cap B(0, R)$ and $V=B(y, r) \cap B(0, R)$, we obtain that for all non-negative measurable functions $f$ and $g$ on $V$ with supports contained in $B(0, R)$,

$$
\begin{aligned}
\left\langle P_{t}^{B(0, R)} f, g\right\rangle \leqslant & \left\langle\mathbb{E} \cdot\left[\mathbb{1}_{\left\{\tau_{B(x, r)} \leqslant t / 2\right\}} P_{t-\tau_{B(x, r)}}^{B(0, R)} f\left(X_{\tau_{B(x, r)}}\right)\right], g\right\rangle \\
& +\left\langle\mathbb{E} \cdot\left[\mathbb{1}_{\left\{\tau_{B(y, r)} \leqslant t / 2\right\}} P_{t-\tau_{B(y, r)}}^{B(0, R)} g\left(X_{\tau_{B(y, r)}}\right)\right], f\right\rangle,
\end{aligned}
$$

where $\langle\cdot, \cdot\rangle$ denotes the inner product on $L^{2}(V ; \mu)$. Let $f$ be supported in $B\left(y, \rho_{0}\right) \cap B(0, R)$ and $g$ be supported in $B\left(x, \rho_{0}\right) \cap B(0, R)$. Then, it holds that

$$
\begin{aligned}
& \left\langle\mathbb{E} .\left[\mathbb{1}_{\left\{\tau_{B(x, r)} \leqslant t / 2\right\}} P_{t-\tau_{B(x, r)}}^{B(0, R)} f\left(X_{\tau_{B(x, r)}}\right)\right], g\right\rangle \\
& =\sum_{z \in B\left(x, \rho_{0}\right) \cap B(0, R)} \mathbb{E}_{z}\left[\mathbb{1}_{\left\{\tau_{B(x, r)} \leqslant t / 2\right\}} P_{t-\tau_{B(x, r)}}^{B(0, R)} f\left(X_{\tau_{B(x, r)}}\right)\right] g(z) \mu_{z} .
\end{aligned}
$$

A similar equality holds for the second term in the right hand side of (2.21).

Below, we write $\tau=\tau_{B(x, r)}$ and $B=B(0, R)$ for simplicity. Set $\rho_{k}=2^{k} \rho_{0}$ for $k \geqslant 1$, and consider the annuli

$$
A_{1}:=B\left(y, \rho_{1}\right), \quad A_{k}:=B\left(y, \rho_{k}\right) \backslash B\left(y, \rho_{k-1}\right), \quad k \geqslant 2 .
$$

Then, for every $z \in B\left(x, \rho_{0}\right)$,

$$
\mathbb{E}_{z}\left[\mathbb{1}_{\{\tau \leqslant t / 2\}} P_{t-\tau}^{B} f\left(X_{\tau}\right)\right]=\sum_{k=1}^{\infty} \mathbb{E}_{z}\left[\mathbb{1}_{\{\tau \leqslant t / 2\}} P_{t-\tau}^{B} f\left(X_{\tau}\right) \mathbb{1}_{\left\{X_{\tau} \in A_{k}\right\}}\right]=: \sum_{k=1}^{\infty} E_{k} .
$$

For $k \geqslant 2$, note that if $X_{\tau} \in A_{k}$, then $\rho\left(X_{\tau}, y\right) \geqslant \rho_{k-1}$. So, for all $v \in B\left(y, \rho_{0}\right)$,

$$
\rho\left(X_{\tau}, v\right) \geqslant \rho_{k-1}-\rho_{0} \geqslant \frac{1}{2} \rho_{k-1}=\frac{1}{4} \rho_{k} .
$$

Recall that, for $\tau \leqslant t / 2$ and $t \geqslant 2 N R^{\theta^{\prime} \alpha}$, it holds that $N R^{\theta^{\prime} \alpha} \leqslant t / 2 \leqslant t-\tau$. Hence, by $\left(H_{q, N}\right)$, if $X_{\tau} \in A_{k}$ for all $k \geqslant 2$, then

$$
P_{t-\tau}^{B} f\left(X_{\tau}\right)=\sum_{v \in B\left(y, \rho_{0}\right)} p^{B}\left(t-\tau, X_{\tau}, v\right) f(v) \mu_{v} \leqslant c t^{-d / \alpha}\left(\frac{t}{\rho_{k}^{\alpha}}\right)^{q}\|f\|_{1} .
$$

According to $\left(H_{0, N}\right)$ and the fact that $\rho_{1}=2 t^{1 / \alpha}$, it is easy to see the inequality above also holds true for $k=1$. 
Next, we separately estimate the terms with $\rho_{k}>r / 2$ and with $\rho_{k} \leqslant r / 2$. Using the facts that $\rho_{0}<r / 4, t / 2 \geqslant N R^{\theta^{\prime} \alpha}$ and $r=\rho(x, y) / 2>4 t^{1 / \alpha} \geqslant 4 R^{\theta^{\prime} \alpha}$, we obtain from (2.10) that for all $z \in B\left(x, \rho_{0}\right) \cap B(0, R) \subseteq B(0, R)$ and $\delta \in(0,1 / 2)$,

$$
\mathbb{P}_{z}(\tau \leqslant t / 2) \leqslant \mathbb{P}_{z}\left(\tau_{B(z, r / 2)} \leqslant t / 2\right) \leqslant c\left(\frac{t}{r^{\alpha}}\right)^{\delta}
$$

Hence, according to all these estimates above, for any $q>0$,

$$
\begin{aligned}
\sum_{k: 2 \rho_{k}>r} E_{k} & \leqslant c \sum_{k: 2 \rho_{k}>r} \mathbb{P}_{z}(\tau \leqslant t / 2) t^{-d / \alpha}\left(\frac{t}{\rho_{k}^{\alpha}}\right)^{q}\|f\|_{1} \leqslant c \sum_{k: 2 \rho_{k}>r}\left(\frac{t}{r^{\alpha}}\right)^{\delta} t^{-d / \alpha}\left(\frac{t}{\rho_{k}^{\alpha}}\right)^{q}\|f\|_{1} \\
& \leqslant c\left(\frac{t}{r^{\alpha}}\right)^{\delta} t^{-d / \alpha}\left(\frac{t}{r^{\alpha}}\right)^{q}\|f\|_{1} \leqslant c t^{-d / \alpha}\left(\frac{t}{r^{\alpha}}\right)^{q+\delta}\|f\|_{1} .
\end{aligned}
$$

When $q=0$,

$$
\sum_{k: 2 \rho_{k}>r} E_{k} \leqslant c t^{-d / \alpha}\|f\|_{1} \mathbb{P}_{z}(\tau \leqslant t / 2) \leqslant c t^{-d / \alpha}\left(\frac{t}{r^{\alpha}}\right)^{\delta}\|f\|_{1} .
$$

On the other hand, for $2 \rho_{k} \leqslant r$, it holds that $2 N^{1 / \alpha} R^{\theta^{\prime}} \leqslant \rho_{1} \leqslant \rho_{k} \leqslant r / 2 \leqslant R$. Then, by (2.18), for all $k \geqslant 1$ and $z \in B\left(x, \rho_{0}\right) \subseteq B(x, r / 2)$,

$$
\mathbb{P}_{z}\left(\tau \leqslant t / 2, X_{\tau} \in A_{k}\right) \leqslant \frac{c t \rho_{k}^{d}}{r^{d+\alpha}} .
$$

Combining this with $\left(H_{q, N}\right)$ yields

$$
\begin{aligned}
\sum_{k: 2 \rho_{k} \leqslant r} E_{k} & \leqslant c \sum_{k: 2 \rho_{k} \leqslant r} \mathbb{P}_{z}\left(\tau \leqslant t / 2, X_{\tau} \in B\left(y, \rho_{k}\right)\right) t^{-d / \alpha}\left(\frac{t}{\rho_{k}^{\alpha}}\right)^{q}\|f\|_{1} \\
& \leqslant c \sum_{k: 2 \rho_{k} \leqslant r} \frac{t \rho_{k}^{d}}{r^{d+\alpha}} \frac{1}{t^{d / \alpha}}\left(\frac{t}{\rho_{k}^{\alpha}}\right)^{q}\|f\|_{1} \leqslant c t^{-d / \alpha} \frac{t^{1+q}}{r^{d+\alpha}}\|f\|_{1} \sum_{k: 2 \rho_{k} \leqslant r} \rho_{k}^{d-\alpha q} \\
& \leqslant c t^{-d / \alpha}\left(\frac{t}{r^{\alpha}}\right)^{1+q}\|f\|_{1},
\end{aligned}
$$

where in the last inequality we used the fact that $\sum_{k: 2 \rho_{k} \leqslant r} \rho_{k}^{d-\alpha q} \leqslant c r^{d-\alpha q}$ due to $q<d / \alpha$.

Thus, according to all the estimates above, we obtain that for any $R>R_{1}, \delta \in(0,1 / 2)$, $t \geqslant 2 N R^{\theta^{\prime} \alpha}, r>4 t^{1 / \alpha}$ and $z \in B\left(x, \rho_{0}\right) \cap B(0, R)$,

$$
\mathbb{E}_{z}\left[\mathbb{1}_{\{\tau \leqslant t / 2\}} P_{t-\tau}^{B} f\left(X_{\tau}\right)\right] \leqslant c t^{-d / \alpha}\left(\frac{t}{r^{\alpha}}\right)^{q+\delta}\|f\|_{1}
$$

and so

$$
\left\langle\mathbb{E} . \mathbb{1}_{\{\tau \leqslant t / 2\}} P_{t-\tau}^{B} f\left(X_{\tau}\right), g\right\rangle \leqslant c t^{-d / \alpha}\left(\frac{t}{r^{\alpha}}\right)^{q+\delta}\|f\|_{1}\|g\|_{1} .
$$

Estimating similarly the second term in the right hand side of $(2.21)$, we finally get that for all $R>R_{1}, \delta \in(0,1 / 2), r>4 t^{1 / \alpha}$ and $t \geqslant 2 N R^{\theta^{\prime} \alpha}$,

$$
\left\langle P_{t}^{B} f, g\right\rangle \leqslant c t^{-d / \alpha}\left(\frac{t}{r^{\alpha}}\right)^{q+\delta}\|f\|_{1}\|g\|_{1},
$$

which yields that $\left(H_{q+\delta, 2 N}\right)$ holds. So (i) has been shown.

Now we turn to (ii). Similarly, it suffices to consider the case $r>4 t^{1 / \alpha}$. Suppose that $\left(H_{q, N}\right)$ holds for some $q>d / \alpha$ and $N \geqslant 1$. Then, following the argument above and 
carefully tracking the constants, we arrive at that for all $R>R_{1}, \delta \in(0,1 / 2), r>4 t^{1 / \alpha}$ and $t \geqslant 2 N R^{\theta^{\prime} \alpha}$,

$$
\sum_{k: 2 \rho_{k}>r} E_{k} \leqslant c t^{-d / \alpha}\left(\frac{t}{r^{\alpha}}\right)^{q+\delta}\|f\|_{1}
$$

and

$$
\sum_{k: 2 \rho_{k} \leqslant r} E_{k} \leqslant c t^{-d / \alpha} \frac{t^{1+q}}{r^{d+\alpha}}\|f\|_{1} \sum_{k: 2 \rho_{k} \leqslant r} \rho_{k}^{d-\alpha q} \leqslant c t^{-d / \alpha}\left(\frac{t}{r^{\alpha}}\right)^{1+(d / \alpha)}\|f\|_{1},
$$

where in the last inequality we used the fact $\sum_{k: 2 \rho_{k} \leqslant r} \rho_{k}^{d-\alpha q} \leqslant c \rho_{0}^{d-\alpha q} \leqslant c t^{d / \alpha-q}$, thanks to $q>d / \alpha$. These estimates together imply that when $q>d / \alpha$, for all $R>R_{1}, \delta \in(0,1 / 2)$, $r>4 t^{1 / \alpha}$ and $t \geqslant 2 N R^{\theta^{\prime} \alpha}$,

$$
\left\langle\mathbb{E} . \mathbb{1}_{\{\tau \leqslant t / 2\}} P_{t-\tau}^{B} f\left(X_{\tau}\right), g\right\rangle \leqslant c t^{-d / \alpha}\left(\frac{t}{r^{\alpha}}\right)^{(1+(d / \alpha)) \wedge(q+\delta)}\|f\|_{1}\|g\|_{1} .
$$

To estimate the second term in the right hand side of (2.21) similarly, we know that for all $R>R_{1}, \delta \in(0,1 / 2), r>4 t^{1 / \alpha}$ and $t \geqslant 2 N R^{\theta^{\prime} \alpha}$,

$$
\left\langle P_{t}^{B} f, g\right\rangle \leqslant c t^{-d / \alpha}\left(\frac{t}{r^{\alpha}}\right)^{(1+(d / \alpha)) \wedge(q+\delta)}\|f\|_{1}\|g\|_{1} .
$$

So $\left(H_{(1+(d / \alpha)) \wedge(q+\delta)), 2 N}\right)$ holds. Thus, we prove (ii) and so the proof is complete.

By using Proposition 2.6, we can establish the following off diagonal upper bounds for heat kernel $p(t, x, y)$.

Proposition 2.7. (Off diagonal upper bounds for the heat kernel) Suppose that Assumptions ( $d$-Vol), (HK1) and (HK2) hold with $\theta \in(0, \alpha /(2 d+\alpha))$ and $R_{0} \geqslant 1$. Then, for every $\theta^{\prime} \in(\theta, \alpha /(2 d+\alpha))$, there is a constant $R_{1} \geqslant 1$ such that for any $x, y \in V$ and $t>0$ with

$$
\rho(x, y) \geqslant\left(R_{1} \vee \rho(0, x) \vee \rho(0, y)\right)^{\alpha\left(1+\theta^{\prime}\right) /(2(d+\alpha))}
$$

and

$$
\rho(x, y)^{2 \theta^{\prime}(d+\alpha) /\left(1+\theta^{\prime}\right)} \leqslant t \leqslant \rho(x, y)^{2(d+\alpha)}\left(R_{1} \vee \rho(0, x) \vee \rho(0, y)\right)^{-\alpha},
$$

we have

$$
p(t, x, y) \leqslant C_{1}\left(1 \vee \mu_{y}^{-1}\right) \frac{t}{\rho(x, y)^{d+\alpha}},
$$

where $C_{1}>0$ is a positive constant independent of $R_{0}, R_{1}, R, x, y$ and $t$.

Proof. Similar to the proof of Proposition 2.3, we apply the Dynkin-Hunt formula and obtain that for every $N>R \geqslant 1$ and $x, y \in B(0, R)$,

$$
\begin{aligned}
p(t, x, y) & =p^{B(0, N)}(t, x, y)+\mathbb{E}_{x}\left[p\left(t-\tau_{B(0, N)}, X_{\left.\tau_{B(0, N)}, y\right)} \mathbb{1}_{\left\{t>\tau_{B(0, N)}\right\}}\right]\right. \\
& =: J_{1, N}(t)+J_{2, N}(t) .
\end{aligned}
$$

According to (2.20) and (2.10), for any $\varepsilon \in(0,1 / 2)$ and $\theta_{1} \in\left(\theta, \theta^{\prime}\right)$, there exists a constant $R_{1} \geqslant 1$ such that for every $N \geqslant 2 R>2 R_{1}, x, y \in B(0, R)$ and $t \geqslant N^{\theta_{1} \alpha}$,

$$
J_{1, N}(t) \leqslant \frac{c_{1} t}{\rho(x, y)^{d+\alpha}}
$$

and

$$
J_{2, N}(t) \leqslant \mu_{y}^{-1} \mathbb{P}_{x}\left(\tau_{B(0, N)}<t\right) \leqslant \mu_{y}^{-1} \mathbb{P}_{x}\left(\tau_{B(x, N / 2)}<t\right) \leqslant c_{2} \mu_{y}^{-1}\left(t N^{-\alpha}\right)^{(1 / 2)-\varepsilon},
$$

where in the inequality above we used again the facts that $p(t, x, y) \leqslant \mu_{y}^{-1}$ for all $x, y \in V$, and $B(x, N / 2) \subset B(0, N)$ for any $x \in B(0, R)$ with $2 R \leqslant N$. 
Set $N(t, x, y):=\left[t^{-1 /(\alpha(1-2 \varepsilon))} \rho(x, y)^{2(d+\alpha) /(\alpha(1-2 \varepsilon))}\right]$. Let $\theta^{\prime} \in(\theta, \alpha /(2 d+\alpha))$ and $\theta_{1} \in$ $\left(\theta, \theta^{\prime}\right)$. For any $x, y \in B(0, R)$ and $t>0$ with $\rho(x, y) \geqslant(3 R)^{\alpha\left(1+\theta^{\prime}\right) /(2(d+\alpha))}$ and

$$
\rho(x, y)^{2 \theta^{\prime}(d+\alpha) /\left(1+\theta^{\prime}\right)} \leqslant t \leqslant \rho(x, y)^{2(d+\alpha)}(3 R)^{-\alpha},
$$

we can choose $\varepsilon>0$ such that

$$
\frac{\theta^{\prime}}{1+\theta^{\prime}}>\frac{\theta_{1}}{1-2 \varepsilon+\theta_{1}}
$$

and so $N(t, x, y) \geqslant 2 R$ and $t \geqslant N(t, x, y)^{\theta_{1} \alpha}$ for $R$ large enough. Note that $\rho(x, y) \geqslant$ $(3 R)^{\alpha\left(1+\theta^{\prime}\right) /(2(d+\alpha))}$ implies $\rho(x, y)^{2(d+\alpha)}(3 R)^{-\alpha} \geqslant \rho(x, y)^{2 \theta^{\prime}(d+\alpha) /\left(1+\theta^{\prime}\right)}$. Then, applying $N=N(t, x, y)$ into $(2.23)$ and $(2.24)$, we can obtain that for any $x, y \in B(0, R)$ and $t>0$ with $\rho(x, y) \geqslant(3 R)^{\alpha\left(1+\theta^{\prime}\right) /(2(d+\alpha))}$ and $\rho(x, y)^{2 \theta^{\prime}(d+\alpha) /\left(1+\theta^{\prime}\right)} \leqslant t \leqslant \rho(x, y)^{2(d+\alpha)}(3 R)^{-\alpha}$, it holds

$$
p(t, x, y) \leqslant J_{1, N(t, x, y)}(t)+J_{2, N(t, x, y)}(t) \leqslant \frac{c_{3}\left(1 \vee \mu_{y}^{-1}\right) t}{\rho(x, y)^{d+\alpha}},
$$

where in the last inequality we used the fact that

$$
N \geqslant t^{-(1+2 \varepsilon) /(\alpha(1-2 \varepsilon))} \rho(x, y)^{2(d+\alpha) /(\alpha(1-2 \varepsilon))} .
$$

Therefore, we prove the second desired estimates in (2.22) by taking $R=R_{1} \vee \rho(0, x) \vee$ $\rho(0, y)$ and $\theta^{\prime}$ a little bit larger.

Finally, according to Propositions 2.3 and 2.7, we can summarize the following upper bound for the heat kernel $p(t, x, y)$.

Theorem 2.8. (Upper bound for the heat kernel) Suppose Assumptions ( $d$-Vol), (HK1) and (HK2) hold with $\theta \in(0, \alpha /(2 d+\alpha))$ and $R_{0} \geqslant 1$. Then, for every $\theta^{\prime} \in\left(\theta_{0}, 1\right)$ with $\theta_{0}:=\max \{2 \theta(d+\alpha) /(\alpha(1+\theta)), \alpha /(2 d+\alpha)\}$, there is a constant $R_{1} \geqslant 1$ such that for any $R>R_{1}, x, y \in V$ with $t>\left(R_{1} \vee \rho(0, x) \vee \rho(0, y)\right)^{\theta^{\prime} \alpha}$,

$$
p(t, x, y) \leqslant C_{1}\left(1 \vee \mu_{y}^{-1}\right)\left(t^{-d / \alpha} \wedge \frac{t}{\rho(x, y)^{d+\alpha}}\right),
$$

where $C_{1}>0$ is independent of $R_{0}, R_{1}, R, x, y$ and $t$.

Proof. For any $x, y \in V$, let $D(x, y)=R_{1} \vee \rho(0, x) \vee \rho(0, y)$. We first consider the case that $\rho(x, y) \geqslant D(x, y)^{\alpha /(2 d+\alpha)}$. Below, we set $\theta_{0}^{\prime}=2 \theta(d+\alpha) /(\alpha(1+\theta))$. Note that, for any $\theta^{\prime} \in\left(\theta_{0}^{\prime}, 1\right)$, we can find $\theta_{1} \in(\theta, \alpha /(2 d+\alpha))$ such that $\theta^{\prime}=2 \theta_{1}(d+\alpha) /\left(\alpha\left(1+\theta_{1}\right)\right)$. It follows from the facts $\theta_{1}<\alpha /(2 d+\alpha)$ and $D(x, y)^{\alpha /(2 d+\alpha)} \leqslant \rho(x, y) \leqslant 2 D(x, y)$ that $\rho(x, y) \geqslant D(x, y)^{\alpha\left(1+\theta_{1}\right) /(2(d+\alpha))}$, and

$$
\rho(x, y)^{2 \theta_{1}(d+\alpha) /\left(1+\theta_{1}\right)}=\rho(x, y)^{\theta^{\prime} \alpha} \leqslant(2 D(x, y))^{\theta^{\prime} \alpha},
$$

as well as

$$
\rho(x, y)^{2(d+\alpha)} D(x, y)^{-\alpha} \geqslant \rho(x, y)^{\alpha} .
$$

These along with $(2.22)$ yield that for any $D(x, y)^{\theta^{\prime} \alpha} \leqslant t \leqslant \rho(x, y)^{\alpha}$ (increasing $\theta^{\prime}$ a little larger if necessary),

$$
p(t, x, y) \leqslant \frac{c_{2}\left(1 \vee \mu_{y}^{-1}\right) t}{\rho(x, y)^{d+\alpha}} .
$$

On the other hand, note that $\theta<\theta_{0}^{\prime}<\theta^{\prime}$. According to (2.14),

$$
p(t, x, y) \leqslant c_{3}\left(1 \vee \mu_{y}^{-1}\right) t^{-d / \alpha}, \quad t>D(x, y)^{\theta^{\prime} \alpha} .
$$

Combing both estimates above yields $(2.25)$ for the case that $\rho(x, y) \geqslant D(x, y)^{\alpha /(2 d+\alpha)}$.

Next, we consider the case that $\rho(x, y) \leqslant D(x, y)^{\alpha /(2 d+\alpha)}$. Since $\theta^{\prime}>\theta_{0} \geqslant \alpha /(2 d+\alpha)$, it holds that

$$
\rho(x, y) \leqslant D(x, y)^{\alpha /(2 d+\alpha)} \leqslant D(x, y)^{\theta^{\prime}} .
$$


Hence, (2.25) follows from (2.26) for the case that $\rho(x, y) \leqslant D(x, y)^{\alpha /(2 d+\alpha)}$. Therefore, we prove the desired assertion.

Remark 2.9. According to the proofs above, the uniform pointwise upper bound (1.2) of $\mu_{x}$ is only used to derive Theorem 2.2, see [24, Page 13, line 7-8] for the argument of the assertion that $M(t) \geqslant 1 / 2$. In fact, for this assertion (1.2) in Assumption ( $d$-Vol) can be replaced by the condition that there exists a constant $R_{0} \geqslant 1$ such that

$$
\sup _{x \in B(0, R)} \mu_{x} \leqslant c_{\mu} R^{\theta d}, \quad \forall R \geqslant R_{0},
$$

where $\theta \in(0, \alpha /(2 d+\alpha))$ is the constant in Assumption (HK1), and $c_{\mu}>0$ is independent of $R_{0}$ and $R$. Different from nearest neighbor models, some priori estimates of heat kernel (see e.g. [8]) are not available, and the maximum principle (see e.g. [10]) does not work in our setting. We believe that some kind of upper bounds for $\mu_{x}$ are required. On the other hand, we also note that, similar to nearest neighbor models (see [10, (1.5)] and [22, Assumption 1.1(v)]), we need some control on the lower bounds of $\mu_{x}$, see (1.3).

2.3. Lower bounds for the heat kernel estimates. Let $Z:=\left(Z_{t}\right)_{t \geqslant 0}=\left(U_{t}, X_{t}\right)_{t \geqslant 0}$ be the time-space process such that $U_{t}=U_{0}+t$ for any $t \geqslant 0$. We say that a measurable function $q(t, x)$ on $[0, \infty) \times V$ is parabolic in an open subset $A$ of $[0, \infty) \times V$, if for every relatively compact open subset $A_{1}$ of $A, q(t, x)=\mathbb{E}^{(t, x)} q\left(Z_{\tau_{A_{1}}}\right)$ for every $(t, x) \in A_{1}$.

Let $C_{0}>0$ be the constant in (2.9). For every $t \geqslant 0, R \geqslant 1$ and $x, y \in V$, set $Q(t, x, R)=\left(t, t+C_{0} R^{\alpha}\right) \times B(x, R)$.

Theorem 2.10. Suppose that Assumptions ( $d$-Vol) and (HK1) hold with $\theta \in(0,1)$ and $R_{0} \geqslant 1$. Then, there exist constants $\delta \in(\theta, 1)$ and $R_{1} \geqslant 1$ such that for all $R>R_{1}$, $x_{0} \in B(0, R), R^{\delta} \leqslant r \leqslant R, t_{0} \geqslant 0$ and parabolic function $q$ on $Q\left(t_{0}, x_{0}, 2 r\right)$,

$$
|q(s, x)-q(t, y)| \leqslant C_{1}\|q\|_{\infty, r}\left(\frac{|t-s|^{1 / \alpha}+\rho(x, y)}{r}\right)^{\beta},
$$

holds for all $(s, x),(t, y) \in Q\left(t_{0}, x_{0}, r\right)$ such that $\left(C_{0}^{-1}|s-t|\right)^{1 / \alpha}+\rho(x, y) \geqslant 2 r^{\delta}$, where $\|q\|_{\infty, r}=\sup _{(s, x) \in\left[t_{0}, t_{0}+C_{0}(2 r)^{\alpha}\right] \times V} q(s, x)$, and $C_{1}>0$ and $\beta \in(0,1)$ are constants independent of $R_{0}, R_{1}, x_{0}, t_{0}, R, r, s, t, x$ and $y$.

Proof. According to Theorem 2.2, we can follow exactly the same argument of [24, Theorem 3.8] to obtain the desired assertion. The details are omitted here.

We also note that, according to (1.4), there exist constants $R_{2} \geqslant 1$ and $c^{*}>0$ (independent of $\left.R_{0}\right)$ such that for every $R>R_{2}, x \in B(0,6 R)$ and $N \geqslant 1$,

$$
\left\{z \in V: N R \leqslant \rho(z, x) \leqslant c^{*} N R\right\} \neq \emptyset .
$$

Proposition 2.11. (Lower bound for the Dirichlet heat kernel) Suppose that Assumptions $(d-\mathbf{V o l}),(\mathbf{H K} 1)$ and $(\mathbf{H K} 3)$ hold with $\theta \in(0,1)$ and $R_{0} \geqslant 1$. Then there exist $R_{1} \geqslant 1, \delta \in(\theta, 1)$ and $C_{1}, C_{2}>0$ (all three are independent of $R_{0}$ and $R_{1}$ ) such that for every $R \geqslant R_{1}, x, y \in B(0, R / 4)$ and $R^{\delta \alpha} \leqslant t \leqslant C_{1} R^{\alpha}$,

$$
p^{B(0, R)}(t, x, y) \geqslant C_{2}\left(t^{-d / \alpha} \wedge \frac{t}{\rho(x, y)^{d+\alpha}}\right) .
$$

Proof. The proof is split into two steps, and the first one is concerned with near-diagonal lower bound estimates.

Step (1) It follows from (2.10) that for each $\theta^{\prime} \in(\theta, 1)$, there exists a constant $\delta \in\left(\theta^{\prime}, 1\right)$ (here we will take $\delta$ a little bit larger such that $\delta \in\left(\theta^{\prime}, 1\right)$ ) and $R_{1} \geqslant 1$ such that for all 
$R \geqslant R_{1}, x \in B(0, R), t \geqslant 2 R^{\theta^{\prime} \alpha}$ and $R^{\delta} \leqslant r \leqslant R$,

$$
\sum_{y \in B(x, r)^{c}} p^{B(0, R)}(t / 2, x, y) \mu_{y} \leqslant \mathbb{P}_{x}\left(\tau_{B(x, r)} \leqslant t / 2\right) \leqslant c_{1}\left(\frac{t}{r^{\alpha}}\right)^{1 / 3} .
$$

Choosing $c_{0}>0$ large enough and $c_{2}>0$ small enough such that $c_{1} c_{0}^{-\alpha / 3} \leqslant 1 / 3, c_{1} c_{2}^{1 / 3} \leqslant$ $1 / 3$ and $c_{0} c_{2}^{1 / \alpha} \leqslant 1 / 2$, we have that for all $R>R_{1}, 2 R^{\theta^{\prime} \alpha} \leqslant t \leqslant c_{2} R^{\alpha}$ and $x \in B(0, R / 2)$,

$$
\begin{aligned}
& \sum_{y \in B\left(x, c_{0} t^{1 / \alpha}\right)} p^{B(0, R)}(t / 2, x, y) \mu_{y} \\
= & \sum_{y \in B(0, R)} p^{B(0, R)}(t / 2, x, y) \mu_{y}-\sum_{y \in B\left(x, c_{0} t^{1 / \alpha}\right)^{c}} p^{B(0, R)}(t / 2, x, y) \mu_{y} \\
= & 1-\mathbb{P}_{x}\left(\tau_{B(0, R)} \leqslant t / 2\right)-\sum_{y \in B\left(x, c_{0} t^{1 / \alpha}\right)^{c}} p^{B(0, R)}(t / 2, x, y) \mu_{y} \\
\geqslant & 1-\mathbb{P}_{x}\left(\tau_{B(x, R / 2)} \leqslant t / 2\right)-\sum_{y \in B\left(x, c_{0} t^{1 / \alpha}\right)^{c}} p^{B(0, R)}(t / 2, x, y) \mu_{y} \\
\geqslant & 1-c_{1}\left(t R^{-\alpha}\right)^{1 / 3}-c_{1} c_{0}^{-\alpha / 3} \sum_{\geqslant} 1-c_{1} c_{2}^{1 / 3}-c_{1} c_{0}^{-\alpha / 3}=: c_{3} \geqslant 1 / 3,
\end{aligned}
$$

where in the first equality we have used the fact that $B\left(x, c_{0} t^{1 / \alpha}\right) \subseteq B(0, R)$, and the second inequality follows from (2.30). By the semigroup property and the Cauchy-Schwarz inequality, we get that for all $R \geqslant R_{1}, x \in B(0, R / 2)$ and $2 R^{\theta^{\prime} \alpha} \leqslant 2 R^{\delta \alpha} \leqslant t \leqslant c_{2} R^{\alpha}$,

$$
\begin{aligned}
p^{B(0, R)}(t, x, x) & =\sum_{y \in B(0, R)} p^{B(0, R)}(t / 2, x, y)^{2} \mu_{y} \geqslant \sum_{y \in B\left(x, c_{0} t^{1 / \alpha}\right)} p^{B(0, R)}(t / 2, x, y)^{2} \mu_{y} \\
& \geqslant c_{4} t^{-d / \alpha}\left(\sum_{y \in B\left(x, c_{0} t^{1 / \alpha}\right)} p^{B(0, R)}(t / 2, x, y) \mu_{y}\right)^{2} \geqslant c_{5} t^{-d / \alpha} .
\end{aligned}
$$

On the other hand, under Assumptions ( $d$-Vol) and (HK1), we have (2.27). Let $C_{0}$ be the constant in (2.27), which is used in the definition of $Q(t, x, R)$. For every fixed $t>0$ and $x \in V$, set

$$
f_{t, x}(s, z)=p^{B(0, R)}(t-s, x, z), \quad(s, z) \in[0, t) \times V .
$$

It is easy to verify that $f_{t, x}(\cdot, \cdot)$ is parabolic on $Q\left(0, x,\left(2^{-1} C_{0}^{-1} t\right)^{1 / \alpha}\right)$ for every $x \in$ $B(0, R / 2)$ and $2 R^{\delta \alpha} \leqslant t \leqslant c_{2} R^{\alpha}$ with some $c_{2}>0$ small enough. Therefore, according to $(2.27)$, there exist $\delta \in(\theta, 1)$ and $R_{1} \geqslant 1$ (for simplicity we adopt the same $R_{1}$ and $\delta$ as those in (2.30)) such that for all $R>R_{1}, 4 R^{\delta \alpha} \leqslant t \leqslant c_{2} R^{\alpha}, x \in B(0, R / 2)$ and $y \in B\left(x, 3^{-1}\left(2^{-1} C_{0}^{-1} t\right)^{1 / \alpha}\right)$ with $\rho(x, y) \geqslant\left(2^{-1} C_{0}^{-1} t\right)^{\delta / \alpha}$,

$$
\begin{aligned}
& \left|p^{B(0, R)}(t, x, y)-p^{B(0, R)}(t, x, x)\right| \\
& =\left|f_{t, x}(0, y)-f_{t, x}(0, x)\right| \leqslant c_{6}\left(\sup _{s \in B(0, t / 2), z \in V}\left|f_{t, x}(s, z)\right|\right)\left(\frac{\rho(x, y)}{t^{1 / \alpha}}\right)^{\beta} \\
& \leqslant c_{6}\left(\sup _{t \geqslant 2 R^{\delta \alpha}, z \in V} p^{B(0, R)}(t, x, z)\right)\left(\frac{\rho(x, y)}{t^{1 / \alpha}}\right)^{\beta} \leqslant c_{7} t^{-d / \alpha}\left(\frac{\rho(x, y)}{t^{1 / \alpha}}\right)^{\beta},
\end{aligned}
$$

where the last inequality is due to (2.3). Combining this with (2.31), we can find constants $c_{8}>0$ and $c_{9}>0$ (small enough satisfying that $c_{7}\left(4 c_{9}\right)^{\beta / \alpha} \leqslant c_{5} / 2$ ) such that for $4 R^{\delta \alpha} \leqslant$ 
$t \leqslant c_{2} R^{\alpha}$ and $x, y \in B(0, R / 2)$ with $c_{8} t^{\delta / \alpha}:=\left(2^{-1} C_{0}^{-1} t\right)^{\delta / \alpha} \leqslant \rho(x, y) \leqslant 4 c_{9} t^{1 / \alpha}$,

$$
\begin{aligned}
p^{B(0, R)}(t, x, y) & \geqslant p^{B(0, R)}(t, x, x)-c_{7} t^{-d / \alpha}\left(\frac{\rho(x, y)}{t^{1 / \alpha}}\right)^{\beta} \\
& \geqslant c_{5} t^{-d / \alpha}-c_{7}\left(2 c_{9}\right)^{\beta / \alpha} t^{-d / \alpha} \geqslant c_{10} t^{-d / \alpha} .
\end{aligned}
$$

Next, we consider the case that $x, y \in B(0, R / 3)$ with $\rho(x, y)<c_{8} t^{\delta / \alpha}$. By (2.28), we can find $z \in B(0, R / 2)$ and $c_{11}>3 c_{8}$ such that $c_{11} t^{\delta / \alpha} \leqslant \rho(z, x) \leqslant c^{*} c_{11} t^{\delta / \alpha}$. Thus, $2 c_{8} t^{\delta / \alpha}<\rho(y, z) \leqslant\left(1+c^{*}\right) c_{11} t^{\delta / \alpha} \leqslant c_{9} t^{1 / \alpha}$ (if we choose $R$ large enough). Then, according to the argument of $(2.32)$, we can obtain that

$$
\left|p^{B(0, R)}(t, x, y)-p^{B(0, R)}(t, x, z)\right| \leqslant c_{7} t^{-d / \alpha}\left(\frac{\rho(y, z)}{t^{1 / \alpha}}\right)^{\beta} \leqslant c_{12} t^{-d / \alpha} t^{-(1-\delta) \beta / \alpha} .
$$

On the other hand, by $(2.33), p^{B(0, R)}(t, x, z) \geqslant c_{10} t^{-d / \alpha}$. Hence, it holds that

$$
\begin{aligned}
p^{B(0, R)}(t, x, y) & \geqslant p^{B(0, R)}(t, x, z)-\left|p^{B(0, R)}(t, x, y)-p^{B(0, R)}(t, x, z)\right| \\
& \geqslant c_{10} t^{-d / \alpha}-c_{12} t^{-d / \alpha} t^{-(1-\delta) \beta / \alpha} \geqslant c_{13} t^{-d / \alpha} .
\end{aligned}
$$

By now we have proved that for all $R>R_{1}, 4 R^{\delta \alpha} \leqslant t \leqslant c_{2} R^{\alpha}$ and $x, y \in B(0, R / 3)$ with $\rho(x, y) \leqslant 4 c_{9} t^{1 / \alpha}$,

$$
p^{B(0, R)}(t, x, y) \geqslant c_{13} t^{-d / \alpha}
$$

Step (2) Now, by the strong Markov property, for all $R>R_{1}, 4 R^{\delta \alpha} \leqslant t \leqslant c_{2} R^{\alpha}$, $a \in(0,1)$ small enough and $x, y \in B(0, R / 4)$ with $\rho(x, y)>4 c_{9} t^{1 / \alpha}$,

$$
\begin{aligned}
& \mathbb{P}_{x}\left(X_{a t} \in B\left(y, 2 c_{9} t^{1 / \alpha}\right) ; \tau_{B(0, R)}>a t\right) \\
& \geqslant \mathbb{P}_{x}\left(\sigma_{B\left(y, c_{9} t^{1 / \alpha}\right)} \leqslant a t ; \sup _{s \in\left[\sigma_{B\left(y, c_{9} t^{1 / \alpha}\right)}, a t\right]} \rho\left(X_{s}, X_{\sigma_{B\left(y, c_{9} t^{1 / \alpha}\right)}}\right)<c_{9} t^{1 / \alpha}\right) \\
& \geqslant \mathbb{P}_{x}\left(\sigma_{B\left(y, c_{9} t^{1 / \alpha}\right)} \leqslant a t\right) \inf _{z \in B\left(y, c_{9} t^{1 / \alpha}\right)} \mathbb{P}_{z}\left(\tau_{B\left(z, c_{9} t^{1 / \alpha}\right)}>a t\right) \\
& \geqslant \mathbb{P}_{x}\left(\sigma_{B\left(y, c_{9} t^{1 / \alpha}\right)} \leqslant a t\right) \inf _{z \in B(0, R)} \mathbb{P}_{z}\left(\tau_{B\left(z, c_{9} t^{1 / \alpha}\right)}>a t\right) \\
& \geqslant \mathbb{P}_{x}\left(\sigma_{B\left(y, c_{9} t^{1 / \alpha}\right)} \leqslant a t\right)\left(1-\frac{c_{1} a t}{\left(c_{9} t^{1 / \alpha}\right)^{\alpha}}\right) \\
& \geqslant \frac{1}{2} \mathbb{P}_{x}\left(X_{(a t) \wedge \tau_{B\left(x, c_{9} t^{1 / \alpha}\right)}} \in B\left(y, c_{9} t^{1 / \alpha}\right)\right) .
\end{aligned}
$$

Here the third inequality above is due to $B\left(y, c_{9} t^{1 / \alpha}\right) \subseteq B(0, R)$ since $y \in B(0, R / 4)$ and $t \leqslant c_{2} R^{\alpha}$ for some $c_{2}$ small enough, and in the fourth inequality we used (2.10) thanks to the facts that $a t \geqslant\left(c_{9} t^{1 / \alpha}\right)^{\delta \alpha}$ and $c_{9} t^{1 / \alpha} \geqslant c_{9} 4^{1 / \alpha} R^{\delta} \geqslant R^{\theta^{\prime \prime}}$ for any $\theta^{\prime \prime} \in\left(\theta^{\prime}, \delta\right)$ and $R$ large enough, and in the last inequality we used the fact that $a$ is small enough such that $a c_{9}^{-\alpha} c_{1} \leqslant 1 / 2$.

Note that $B\left(x, c_{9} t^{1 / \alpha}\right) \cap B\left(y, c_{9} t^{1 / \alpha}\right)=\emptyset$ for any $x, y \in B(0, R / 4)$ with $\rho(x, y) \geqslant 4 c_{9} t^{1 / \alpha}$. Then, by (2.17), for all $R>R_{1}, 4 R^{\theta^{\prime} \alpha} \leqslant t \leqslant c_{2} R^{\alpha}$ and $x, y \in B(0, R / 4)$ with $\rho(x, y) \geqslant$ 
$4 c_{9} t^{1 / \alpha}$, we have

$$
\begin{aligned}
& \mathbb{P}_{x}\left(X_{(a t) \wedge \tau_{B\left(x, c_{9} t^{1 / \alpha}\right)}} \in B\left(y, c_{9} t^{1 / \alpha}\right)\right) \\
& =\mathbb{E}_{x}\left[\sum_{s \leqslant(a t) \wedge \tau_{B\left(x, c_{9} t^{1 / \alpha}\right)}} \mathbb{1}_{\left\{X_{s} \in B\left(y, c_{9} t^{1 / \alpha}\right)\right\}}\right]
\end{aligned}
$$

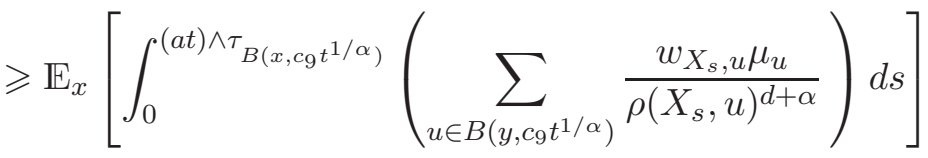

$$
\begin{aligned}
& \geqslant c_{14} \rho(x, y)^{-d-\alpha}\left(\inf _{v \in B\left(x, c_{9} t^{1 / \alpha}\right)} \sum_{u \in B\left(y, c_{9} t^{1 / \alpha}\right)} w_{v, u} \mu_{u}\right) \mathbb{E}_{x}\left[(a t) \wedge \tau_{B\left(x, c_{9} t^{1 / \alpha}\right)}\right] \\
& \geqslant c_{15} \mathbb{P}_{x}\left[\tau_{B\left(x, c_{9} t^{1 / \alpha}\right)} \geqslant a t\right] \frac{t^{1+d / \alpha}}{\rho(x, y)^{d+\alpha}} \geqslant \frac{c_{16} t^{1+d / \alpha}}{\rho(x, y)^{d+\alpha}} \text {. }
\end{aligned}
$$

Here in the second inequality we have used the fact that for any $v \in B\left(x, c_{9} t^{1 / \alpha}\right)$ and $u \in B\left(y, c_{9} t^{1 / \alpha}\right)$,

$$
\rho(v, u) \leqslant \rho(v, x)+\rho(x, y)+\rho(y, u) \leqslant \rho(x, y)+2 c_{9} t^{1 / \alpha} \leqslant 2 \rho(x, y),
$$

in the third inequality we used the fact that $R^{\theta^{\prime}} \leqslant 4^{1 / \alpha} R^{\delta} \leqslant t^{1 / \alpha} \leqslant c_{2}^{1 / \alpha} R \leqslant R$ for $\theta^{\prime} \in$ $(\theta, \delta)$ and Assumption (HK3), and the last inequality follows from the same argument in the fourth inequality of $(2.34)$.

Note again that $B\left(y, c_{9} t^{1 / \alpha}\right) \subseteq B(0, R / 3)$ since $y \in B(0, R / 4)$ and $t \leqslant c_{2} R^{\alpha}$ for some $c_{2}$ small enough. Since $4 R^{\theta^{\prime} \alpha} \leqslant 4 R^{\delta \alpha} \leqslant t \leqslant c_{2} R^{\alpha} \leqslant R^{\alpha}$ for $\theta^{\prime} \in(\theta, \delta)$, we can obtain from (2.33) that for any $a \in(0,1 / 2)$,

$$
\inf _{y \in B(0, R / 3), z \in B\left(y, c_{9} t^{1 / \alpha}\right)} p^{B(0, R)}((1-a) t, y, z) \geqslant c_{17} t^{-d / \alpha} .
$$

Hence, combining this with (2.34) and (2.35), for every $R>R_{1}, 4 R^{\delta \alpha} \leqslant t \leqslant c_{2} R^{\alpha}$ and $x, y \in B(0, R / 4)$ with $\rho(x, y) \geqslant 4 c_{9} t^{1 / \alpha}$,

$$
\begin{aligned}
p^{B(0, R)}(t, x, y) & \geqslant \sum_{z \in B\left(y, c_{9} t^{1 / \alpha}\right)} p^{B(0, R)}(a t, x, z) p^{B(0, R)}((1-a) t, z, y) \mu_{z} \\
& \geqslant \inf _{z \in B\left(y, c_{9} t^{1 / \alpha}\right)} p^{B(0, R)}((1-a) t, z, y) \sum_{z \in B\left(y, c_{9} t^{1 / \alpha}\right)} p^{B(0, R)}(a t, x, z) \mu_{z} \\
& \geqslant c_{18} t^{-d / \alpha} \mathbb{P}_{x}\left(X_{a t} \in B\left(y, 2 c_{9} t^{1 / \alpha}\right), \tau_{B(0, R)}>a t\right) \geqslant \frac{c_{19} t}{\rho(x, y)^{d+\alpha}} .
\end{aligned}
$$

Therefore, by all the estimates above, we prove the desired assertion by change $\delta$ a little large if necessary.

As a consequence of Proposition 2.11, we can obtain the following lower bound of the heat kernel.

Theorem 2.12. (Lower bound for the heat kernel) Suppose that Assumptions ( $d$ Vol), (HK1) and (HK3) hold with $\theta \in(0,1)$ and $R_{0} \geqslant 1$. Then there exist $R_{1} \geqslant 1$ and $\delta \in(\theta, 1)$ (independent of $R_{0}$ and $\left.R_{1}\right)$ such that for every $x, y \in V$ and $t>(\rho(0, x) \vee$ $\left.\rho(0, y) \vee R_{1}\right)^{\delta \alpha}$,

$$
p(t, x, y) \geqslant C_{1}\left(t^{-d / \alpha} \wedge \frac{t}{\rho(x, y)^{d+\alpha}}\right) .
$$


where $C_{1}>0$ is independent of $R_{0}, R_{1}, t, x$ and $y$. In particular, there exist $T_{0}>0$ and $\delta \in(\theta, 1)$ (independent of $R_{0}$ and $T_{0}$ ) such that for all $t \geqslant T_{0}$ and $x, y \in B\left(0, t^{1 /(\delta \alpha)}\right)$, (2.36) holds with $C_{1}>0$ independent of $R_{0}, T_{0}, t, x$ and $y$.

Proof. Let $x, y \in V$ and $t>0$ such that $t \geqslant D(x, y)^{\delta \alpha}$, where $D(x, y)=\rho(0, x) \vee \rho(0, y) \vee R_{1}$ for some large $R_{1} \geqslant 1$ and $\delta \in(\theta, 1)$. Then, taking $R \geqslant D(x, y)$ such that $x, y \in B(0, R)$ and $R^{\delta \alpha} \leqslant t \leqslant C_{1} R^{\alpha}$ with $C_{1}$ being the constant in Proposition 2.11, and applying (2.29) with $t$ and $R$ above, we can get

$$
p(t, x, y) \geqslant p^{B(0, R)}(t, x, y) \geqslant c_{1}\left(t^{-d / \alpha} \wedge \frac{t}{\rho(x, y)^{d+\alpha}}\right) .
$$

This proves (2.36) by choosing $\delta$ a little bit larger. Choosing $R=t^{1 /(\delta \alpha)}$ and then renaming $R_{1}^{\delta \alpha}$ as $T_{0}$ in the estimate above, we can prove the second desired assertion also by taking $\delta$ a little bit larger.

\section{Green Function Estimates and Elliptic Harnack Inequalities}

In this section, we give some estimates of Green functions and then give a consequence of elliptic Harnack inequalities. The results in this section is used in Subsection 4.2.2. Throughout this section, we assume that $(G, \mu)$ satisfies the global d-set condition; that is,

$$
c_{\mu}^{-1} \leqslant \mu_{x} \leqslant c_{\mu}, \quad c_{\mu}^{-1} r^{d} \leqslant \mu(B(x, r)) \leqslant c_{\mu} r^{d}, \quad r \geqslant 1, x \in V .
$$

We also suppose in this section that $w_{x, y}>0$ for all $x, y \in V$ with $x \neq y$.

3.1. Upper bounds for the Dirichlet heat kernel: small times. In this subsection we present some upper bounds of the Dirichlet heat kernel for small times, which are used to obtain estimates for Green functions in the next subsection.

In the nearest neighbor conductance models, some priori estimates are used for the small time heat kernel estimates, see [35, Corollaries 11 and 12] or [40, Theorems 2.1 and 2.2]. However, such estimates are not known for the long range case. In order to overcome this obstacle, we will adopt the localization approach (see [24, Section 2.2] for more details). We note that for our arguments to work, we need the global $d$-set condition (3.1) and the assumption that $w_{x, y}>0$ for all $x, y \in V$ with $x \neq y$. Since finally we will apply results in this part to Subsection 4.2.2, in which we show the elliptic Harnack inequalities do not hold in general on long-range random conductance models, we are satisfied with the assumptions. It is an interesting open problem how much we can relax the assumptions.

For any fixed $R \geqslant 1, \gamma \in(0,1)$ and $x_{0} \in B(0,2 R)$, we define the following symmetric regular Dirichlet form $\left(\hat{D}^{x_{0}, \gamma, R}, \hat{\mathscr{F}} x_{0}, \gamma, R\right)$ :

$$
\begin{aligned}
\hat{D}^{x_{0}, \gamma, R}(f, f) & =\sum_{x, y \in V: \rho(x, y) \leqslant \gamma R}(f(x)-f(y))^{2} \frac{\hat{w}_{x, y}}{\rho(x, y)^{d+\alpha}} \mu_{x} \mu_{y}, \quad f \in \hat{\mathscr{F}}^{x_{0}, \gamma, R}, \\
\hat{\mathscr{F}}^{x_{0}, \gamma, R} & =\left\{f \in L^{2}(V ; \mu): \hat{D}^{x_{0}, \gamma, R}(f, f)<\infty\right\},
\end{aligned}
$$

where

$$
\hat{w}_{x, y}= \begin{cases}w_{x, y}, & \text { if } x \in B\left(x_{0}, R\right) \text { or } y \in B\left(x_{0}, R\right), \\ 1, & \text { otherwise. }\end{cases}
$$

Here we omit the parameters $x_{0}$ and $R$ in the definition of $\hat{w}_{x, y}$ for simplicity. Denote by $\left(\hat{X}_{t}^{x_{0}, \gamma, R}\right)_{t \geqslant 0}$ the symmetric Hunt process associated with $\left(\hat{D}^{x_{0}, \gamma, R}, \hat{\mathscr{F}} x_{0}, \gamma, R\right)$.

For every $r>0$, set

$$
\Theta(r):=1+\sup _{x, y \in B(0, r)} w_{x, y}^{-1}
$$


Lemma 3.1. Suppose that Assumption (HK1) holds with $\theta \in(0,1)$ and $R_{0} \geqslant 1$. Then, for any $\gamma \in(0, \alpha /(3(d+\alpha))]$, there exists a constant $R_{1} \geqslant 1$ such that for every $R>R_{1}$, $x_{0} \in B(0,2 R), R^{\theta} \leqslant r \leqslant R, x \in V$ and $t>0$,

$$
\mathbb{P}_{x}\left(\rho\left(\hat{X}_{t}^{x_{0}, \gamma, r}, x\right)>r\right) \leqslant C_{1} \Theta(4 R)^{d / \alpha} \frac{t}{r^{\alpha}},
$$

where $C_{0}, C_{1}>0$ are independent of $R_{0}, R_{1}, R, r, x_{0}, x$ and $t$ (but may depend on $\gamma$ ).

Proof. We only need to verify (3.3) the case that $0<t \leqslant(\gamma r)^{\alpha} / 2$. The proof is split into two steps.

Step (1) By Assumption (HK1) and the definition of $\hat{w}_{x, y}$, we can verify that for every $1 \leqslant r \leqslant R$ and $x_{0} \in B(0,2 R)$,

$$
\sup _{x \in V} \sum_{y \in V: \rho(x, y) \leqslant c_{*} r} \hat{w}_{x, y}^{-1} \mu_{y} \leqslant c_{0} \Theta(4 R) r^{d}
$$

with $c_{*}:=8 c_{\mu}^{2 / d}$, and also that there is a constant $R_{0} \geqslant 1$ such that for all $R \geqslant R_{0}$, $x_{0} \in B(0,2 R)$ and $R^{\theta} \leqslant r \leqslant R$,

$$
\sup _{x \in V} \sum_{y \in V: \rho(x, y) \leqslant \gamma r} \frac{\hat{w}_{x, y} \mu_{y}}{\rho(x, y)^{d+\alpha-2}} \leqslant c_{0}(\gamma r)^{2-\alpha} .
$$

Using (3.4) and (3.1), and following the argument of (2.4), we can obtain that for any $x_{0} \in B(0,2 R), 1 \leqslant r \leqslant R, x \in V$ and any measurable function $f$ on $V$,

$$
\sum_{z \in B(x, r)}\left(f(z)-(f)_{B(x, r)}\right)^{2} \mu_{z} \leqslant c_{1} \Theta(4 R) r^{\alpha} \sum_{z, y \in B(x, r)}(f(z)-f(y))^{2} \frac{\hat{w}_{z, y}}{\rho(z, y)^{d+\alpha}} \mu_{y} \mu_{z} .
$$

Let $\hat{p}^{x_{0}, \gamma, r}(t, x, y)$ be the heat kernel associated with the process $\left(\hat{X}_{t}^{x_{0}, \gamma, r}\right)_{t \geqslant 0}$, and let $\psi_{x_{0}}(t, x)=\hat{p}^{x_{0}, \gamma, r}(2 t, x, x)=\sum_{z \in V} \hat{p}^{x_{0}, \gamma, r}(t, x, z)^{2} \mu_{z}$ for any $t>0$ and $x \in V$. Then, it follows from (3.6) and the argument of (2.6) that for all $x_{0} \in B(0,2 R), x \in V, 1 \leqslant r \leqslant R$, $0<t \leqslant(\gamma r)^{\alpha} / 2$ and $1 \leqslant r(t) \leqslant \gamma r$,

$$
\psi_{x_{0}}^{\prime}(t, x) \leqslant c_{2} \Theta(4 R)^{-1} r(t)^{-\alpha}\left(\psi_{x_{0}}(t, x)-c_{3} r(t)^{-d}\right) .
$$

Here and in what follows constants $c_{i}$ may depend on $\gamma$. Thus, further following part (3) in the proof of Proposition 2.1, we can finally obtain for all $x_{0} \in B(0,2 R)$ and $1 \leqslant r \leqslant R$,

$$
\hat{p}^{x_{0}, \gamma, r}(t, x, y) \leqslant c_{4} \Theta(4 R)^{d / \alpha} t^{-d / \alpha}, \quad x, y \in V, 0<t \leqslant(\gamma r)^{\alpha} / 2 .
$$

Step (2) For simplicity, in the following we omit the index $x_{0}$. According to (3.7) and [23, Theorem (3.25)], for any $x, y \in V, 1 \leqslant r \leqslant R$ and $0<t \leqslant(\gamma r)^{\alpha} / 2$, we have

$$
\hat{p}^{x_{0}, \gamma, r}(t, x, y) \leqslant c_{4}^{*} \Theta(4 R)^{d / \alpha} t^{-d / \alpha} \inf _{\psi \in L^{\infty}(V ; \mu)} \exp (\psi(x)-\psi(y)+c(\psi) t),
$$

where $c(\psi)=\sup _{x \in V} b(\psi, x)$ and

$$
b(\psi, x)=\sum_{z \in V: \rho(z, x) \leqslant \gamma r} \frac{\hat{w}_{x, z} \mu_{z}}{\rho(x, z)^{d+\alpha}}\left(e^{\psi(z)-\psi(x)}-1\right)^{2} .
$$

Next, for fixed $x, y \in V$ and $\lambda>0$, define

$$
\psi_{\lambda}(z)=\lambda(\rho(x, y) \wedge \rho(z, x)) \in L^{\infty}(V ; \mu) .
$$

Then,

$$
\begin{aligned}
b\left(\psi_{\lambda}, x\right) & \leqslant \sum_{z \in V: \rho(x, z) \leqslant \gamma r} \frac{\hat{w}_{x, z} \mu_{z}}{\rho(x, z)^{d+\alpha}}\left(e^{\lambda(\rho(x, z) \wedge \rho(x, y))}-1\right)^{2} \\
& \leqslant \lambda^{2} e^{2 \lambda \gamma r} \sum_{z \in V: \rho(z, x) \leqslant \gamma r} \hat{w}_{x, z} \mu_{z} \rho(x, z)^{2-d-\alpha}=: J(x),
\end{aligned}
$$


where in the first inequality we used the facts that $s \mapsto\left(e^{s}-1\right)^{2}$ is increasing for $s \geqslant 0$ and $\left|\psi_{\lambda}(z)-\psi_{\lambda}(y)\right| \leqslant \lambda(\rho(y, z) \wedge \rho(x, y))$ for any $x, y, z \in V$, and the second inequality follows from the inequality that $\left|e^{s}-1\right|^{2} \leqslant s^{2} e^{2|s|}$ for $s \geqslant 0$. On the other hand, it is obvious that, by (3.5), we can find a constant $R_{1} \geqslant 1$ such that for all $R>R_{1}, x_{0} \in B(0,2 R)$ and $R^{\theta} \leqslant r \leqslant R$,

$$
\sup _{x \in V} J(x) \leqslant c_{5} e^{2 \lambda \gamma r}(\lambda \gamma r)^{2}(\gamma r)^{-\alpha} .
$$

Combining both estimates above, we arrive at that for all $R>R_{1}, x_{0} \in B(0,2 R)$ and $R^{\theta} \leqslant r \leqslant R$,

$$
\sup _{x \in V} b\left(\psi_{\lambda}, x\right) \leqslant c_{5} e^{3 \lambda \gamma r}(\gamma r)^{-\alpha},
$$

where we have used the fact that $s^{2} e^{2 s} \leqslant 2 e^{3 s}$ for $s \geqslant 0$.

Now we suppose that $R>R_{1}, x_{0} \in B(0,2 R)$ and $R^{\theta} \leqslant r \leqslant R$. Hence, for any $0<t \leqslant(\gamma r)^{\alpha} / 2$ and $x, y \in V$,

$$
\hat{p}^{\gamma, r}(t, x, y) \leqslant c_{4}^{*} \Theta(4 R)^{d / \alpha} t^{-d / \alpha} \exp \left(-\lambda \rho(x, y)+c_{5}(\gamma r)^{-\alpha} e^{3 \lambda \gamma r} t\right) .
$$

Set

$$
\lambda=\frac{1}{3 \gamma r} \log \left(\frac{(\gamma r)^{\alpha}}{t}\right)
$$

in the right side of the inequality above, we get for all $0<t \leqslant(\gamma r)^{\alpha} / 2$ and $x, y \in V$,

$$
\hat{p}^{\gamma, r}(t, x, y) \leqslant c_{6} \Theta(4 R)^{d / \alpha} t^{-d / \alpha}\left(\frac{t}{(\gamma r)^{\alpha}}\right)^{\rho(x, y) /(3 \gamma r)} .
$$

Therefore, for all $x \in V$ and $0<t \leqslant(\gamma r)^{\alpha} / 2$,

$$
\begin{aligned}
\mathbb{P}_{x}\left(\rho\left(\hat{X}_{t}^{x_{0}, \gamma, r}, x\right)>r\right) & =\sum_{y \in V: \rho(x, y)>r} \hat{p}^{\gamma, r}(t, x, y) \mu_{y} \\
& \leqslant c_{\gamma} \Theta(4 R)^{d / \alpha} t^{-d / \alpha} \sum_{k=0}^{\infty} \sum_{y \in V: 2^{k} r<\rho(x, y) \leqslant 2^{k+1} r}\left(\frac{t}{(\gamma r)^{\alpha}}\right)^{\rho(x, y) /(3 \gamma r)} \\
& \leqslant c_{8} \Theta(4 R)^{d / \alpha} t^{-d / \alpha} \sum_{k=0}^{\infty} \mu\left(B\left(x, 2^{k+1} r\right)\right)\left(\frac{t}{(\gamma r)^{\alpha}}\right)^{2^{k} r /(3 \gamma r)} \\
& \leqslant c_{9} \Theta(4 R)^{d / \alpha} t^{-d / \alpha} \sum_{k=0}^{\infty} 2^{(k+1) d} r^{d}\left(\frac{t}{(\gamma r)^{\alpha}}\right)^{2^{k} /(3 \gamma)} \\
& \leqslant c_{10} \Theta(4 R)^{d / \alpha}\left(\frac{t}{(\gamma r)^{\alpha}}\right)^{(1 /(3 \gamma))-(d / \alpha)} .
\end{aligned}
$$

Taking $\gamma \leqslant \alpha /(3(d+\alpha)$ ) (in particular, $(1 /(3 \gamma))-(d / \alpha) \geqslant 1)$ in the last inequality immediately yields the desired assertion (3.3).

By Lemma 3.1, we can further establish the following estimate for exit time of the process $X$.

Lemma 3.2. Suppose that Assumption (HK1) holds with some constant $\theta \in(0,1)$ and $R_{0} \geqslant 1$. Then for every $\theta^{\prime} \in(\theta, 1)$, there exist constants $R_{1} \geqslant 1$ and $C_{0}, C_{1}>0$ (which are independent of $R_{0}$ and $\left.R_{1}\right)$ such that for every $R>R_{1}, x \in B(0,2 R), R^{\theta^{\prime}} \leqslant r \leqslant 2 R$ and $t>0$,

$$
\mathbb{P}_{x}\left(\tau_{B(x, r)} \leqslant t\right) \leqslant C_{1} \Theta(4 R)^{d / \alpha} \frac{t}{r^{\alpha}}
$$


Proof. It suffices to verify the desired assertion for the case that $0<t \leqslant r^{\alpha} / 2$. The proof is split into two steps.

Step (1) We suppose that $\gamma \in(0, \alpha /(3(d+\alpha))], R \geqslant R_{1}$ for some $R_{1}$ large enough, $x \in B(0,2 R), R^{\theta} \leqslant r / 2 \leqslant R$ and $0<t \leqslant r^{\alpha} / 2$. According to (3.3),

$$
\sup _{s \in[t, 2 t]} \sup _{y \in V} \mathbb{P}_{y}\left(\rho\left(\hat{X}_{s}^{x, \gamma, r}, y\right)>\frac{r}{2}\right) \leqslant c_{1} \Theta(4 R)^{d / \alpha} \frac{t}{r^{\alpha}} .
$$

Hence, by the strong Markov property,

$$
\begin{aligned}
\mathbb{P}_{x}\left(\hat{\tau}_{B(x, r)}^{x, \gamma, r} \leqslant t\right) & \leqslant \mathbb{P}_{x}\left(\hat{\tau}_{B(x, r)}^{x, \gamma, r} \leqslant t, \rho\left(\hat{X}_{2 t}^{x, \gamma, r}, x\right) \leqslant \frac{r}{2}\right)+\mathbb{P}_{x}\left(\rho\left(\hat{X}_{2 t}^{x, \gamma, r}, x\right)>\frac{r}{2}\right) \\
& \leqslant \mathbb{E}_{x}\left[\mathbb{1}_{\left\{\hat{\tau}_{B(x, r)}^{x, \gamma, r} \leqslant t\right\}} \mathbb{P}_{\hat{X}_{\hat{\tau}_{B(x, r)}^{x, \gamma, r}}^{x, r, r}}\left(\rho\left(\hat{X}_{2 t-\hat{\tau}_{B(x, r)}^{x, \gamma, r}}^{x, r, r}, \hat{X}_{0}^{x, \gamma, r}\right)>\frac{r}{2}\right)\right]+c_{1} \Theta(4 R)^{d / \alpha} \frac{t}{r^{\alpha}} \\
& \leqslant \sup _{y \in V} \sup _{s \in[t, 2 t]} \mathbb{P}_{y}\left(\rho\left(\hat{X}_{s}^{x, \gamma, r}, y\right)>\frac{r}{2}\right)+c_{1} \Theta(4 R)^{d / \alpha} \frac{t}{r^{\alpha}} \\
& \leqslant 2 c_{1} \Theta(4 R)^{d / \alpha} \frac{t}{r^{\alpha}}
\end{aligned}
$$

where $\hat{\tau}_{A}^{x, \gamma, r}:=\inf \left\{t>0 ; \hat{X}_{t}^{x, \gamma, r} \notin A\right\}$ is the first exit time from $A \subset V$ for the process $\left(\hat{X}_{t}^{x, \gamma, r}\right)_{t \geqslant 0}$.

Step (2) Similar to the argument in [24, Section 3.1], we define the following Dirichlet form $\left(\hat{D}^{x, r}, \hat{\mathscr{F}}^{x, r}\right)$

$$
\begin{aligned}
\hat{D}^{x, r}(f, f) & =\sum_{y, z \in V}(f(y)-f(z))^{2} \frac{\hat{w}_{y, z}}{\rho(y, z)^{d+\alpha}} \mu_{y} \mu_{z}, \quad f \in \hat{\mathscr{F}}^{x, r}, \\
\hat{\mathscr{F}}^{x, r} & =\left\{f \in L^{2}(V ; \mu): \hat{D}^{x, r}(f, f)<\infty\right\},
\end{aligned}
$$

where $\hat{w}_{x, y}$ is defined by (3.2) with $x_{0}$ and $R$ replaced by $x$ and $r$ respectively. In particular, by the definition of $\left(\hat{D}^{x, r}, \hat{\mathscr{F}}^{x, r}\right)$, we have immediately

$$
\mathbb{P}_{x}\left(\tau_{B(x, r)} \leqslant t\right)=\mathbb{P}_{x}\left(\hat{\tau}_{B(x, r)}^{x, r} \leqslant t\right), \quad x \in V, r, t>0,
$$

where $\left(\hat{X}_{t}^{x, r}\right)_{t \geqslant 0}$ is the Hunt process associated with $\left(\hat{D}^{x, r}, \hat{\mathscr{F}} x, r\right)$, and $\hat{\tau}_{A}^{x, r}:=\inf \{t>0$ : $\left.\hat{X}_{t}^{x, r} \notin A\right\}$ for a subset $A \subset V$. Furthermore, according to [24, Lemma 3.1], we find that

$$
\mathbb{P}_{x}\left(\hat{\tau}_{B(x, r)}^{x, r} \leqslant t\right) \leqslant \mathbb{P}_{x}\left(\hat{\tau}_{B(x, r)}^{x, \gamma, r} \leqslant t\right)+c_{2} t \sup _{y \in B(x, r)} \sum_{z \in V: \rho(y, z)>\gamma r} \frac{w_{y, z} \mu_{z}}{\rho(y, z)^{d+\alpha}}
$$

Noting that $\gamma r>\gamma R^{\theta^{\prime}} \geqslant R^{\theta}$ for $R$ large enough, Assumption (HK1) implies

$$
\sup _{y \in B(x, r)} \sum_{z \in V: \rho(y, z)>\gamma r} \frac{w_{y, z} \mu_{z}}{\rho(y, z)^{d+\alpha}} \leqslant c_{3} r^{-\alpha} .
$$

Combining all the estimates above yields the desired conclusion (3.8).

Now, we are in the position to present the upper bound of the Dirichlet heat kernel for small times.

Proposition 3.3. Suppose that Assumptions (HK1) and (HK2) hold with constants $\theta \in(0,1)$ and $R_{0} \geqslant 1$. Then, for each $\theta^{\prime} \in(\theta, 1)$, there is a constant $R_{1} \geqslant 1$ such that for all $R>R_{1}, x, y \in B(0, R)$ with $\rho(x, y) \geqslant R^{\theta^{\prime}}$, and $0<t \leqslant R^{\alpha}$,

$$
p^{B(0, R)}(t, x, y) \leqslant C_{1} \Theta(4 R)^{k}\left(t^{-d / \alpha} \wedge \frac{t}{\rho(x, y)^{d+\alpha}}\right),
$$

where $C_{0}, C_{1}>0$ and $k \geqslant d / \alpha$ are independent of $R_{0}, R_{1}, R, x, y$ and $t$. 
Proof. We only sketch the proof. First, we note that (3.6) holds with $\hat{w}_{z, y}$ replaced by $w_{z, y}$ and for all $x \in B(0, R)$. Following the proof of Proposition 2.1 and carefully tracking the dependence on $\Theta(4 R)$, we can obtain that there are constants $R_{1} \geqslant 1$ such that for all $R>R_{1}, x, y \in B(0, R)$ and $0<t \leqslant R^{\alpha}$,

$$
p^{B(0, R)}(t, x, y) \leqslant c_{2} \Theta(4 R)^{d / \alpha} t^{-d / \alpha} .
$$

Second, using (3.10), (3.8) (which requires that $x, y \in B(0, R)$ with $\rho(x, y) \geqslant R^{\theta^{\prime}}$ ) and Assumption (HK2), and repeating the argument of Proposition 2.6, we can obtain the desired conclusion. (We note that in the proof of Proposition 2.6, we apply the induction procedure. Hence the term $\Theta(4 R)$ will be multiplied for several times.)

3.2. Estimates for Green functions. The aim of this part is to obtain some estimates for Green functions.

Proposition 3.4. Let $d>\alpha$. Suppose that Assumptions (HK1), (HK2) and (HK3) hold with constants $\theta \in(0,1)$ and $R_{0} \geqslant 1$. Then, for every $\theta^{\prime} \in(\theta, 1)$, there exist constants $R_{1} \geqslant 1$ and $k \geqslant d / \alpha$ such that the following hold for every $R \geqslant R_{1}$,

(1) for any $x, y \in B(0, R)$ with $\rho(x, y) \geqslant R^{\theta^{\prime}}$,

$$
G^{B(0, R)}(x, y) \leqslant C_{1} \Theta(4 R)^{k} \rho(x, y)^{-d+\alpha} ;
$$

(2) for $x, y \in B(0, R / 4)$,

$$
G^{B(0, R)}(x, y) \geqslant C_{2}\left(\rho(x, y)^{-d+\alpha} \wedge R^{-\theta^{\prime}(d-\alpha)}\right),
$$

where $C_{0}, C_{1}, C_{2}, k$ are positive constants independent of $R_{1}, R, x$ and $y$.

Proof. According to (2.3) and (3.9), under Assumptions (HK1) and (HK2) there exist constants $R_{1} \geqslant 1$ and $k \geqslant d / \alpha$ such that for all $R \geqslant R_{1}$ and $x, y \in B(0, R)$ with $\rho(x, y) \geqslant$ $R^{\theta^{\prime}}$,

$$
p^{B(0, R)}(t, x, y) \leqslant \begin{cases}c_{1} \Theta(4 R)^{k} t \rho(x, y)^{-d-\alpha}, & 0<t \leqslant \rho(x, y)^{\alpha}, \\ c_{1} t^{-d / \alpha}, & t>\rho(x, y)^{\alpha} \geqslant R^{\theta^{\prime} \alpha},\end{cases}
$$

which implies that

$$
G^{B(0, R)}(x, y) \leqslant c_{1}\left[\int_{0}^{\rho(x, y)^{\alpha}} \frac{\Theta(4 R)^{k} t}{\rho(x, y)^{d+\alpha}} d t+\int_{\rho(x, y)^{\alpha}}^{\infty} t^{-d / \alpha} d t\right] \leqslant c_{2} \Theta(4 R)^{k} \rho(x, y)^{-d+\alpha},
$$

where in the last inequality we used $d>\alpha$. This proves (3.11).

On the other hand, by (2.29), under Assumptions (HK1) and (HK3) there exists a constant $R_{2} \geqslant 1$ such that for all $R \geqslant R_{2}, x, y \in B(0, R / 4)$ and $R^{\theta^{\prime} \alpha} \leqslant t \leqslant c_{3} R^{\alpha}$,

$$
p^{B(0, R)}(t, x, y) \geqslant c_{4}\left(t^{-d / \alpha} \wedge \frac{t}{\rho(x, y)^{d+\alpha}}\right) .
$$

If $x, y \in B(0, R / 4)$ such that $\rho(x, y) \leqslant R^{\theta^{\prime}}$, then $\rho(x, y) \leqslant t^{1 / \alpha}$ for any $R^{\theta^{\prime} \alpha} \leqslant t \leqslant c_{3} R^{\alpha}$, and so

$$
G^{B(0, R)}(x, y) \geqslant \int_{R^{\theta^{\prime} \alpha}}^{c_{3} R^{\alpha}} p^{B(0, R)}(t, x, y) d t \geqslant c_{4} \int_{R^{\theta^{\prime} \alpha}}^{c_{3} R^{\alpha}} t^{-d / \alpha} d t \geqslant c_{5} R^{-\theta^{\prime}(d-\alpha)} .
$$

While for any $x, y \in B(0, R / 4)$ with $\rho(x, y)>R^{\theta^{\prime}}$, it holds

$$
\begin{aligned}
G^{B(0, R)}(x, y) & \geqslant c_{4}\left(\int_{R^{\theta^{\prime} \alpha}}^{\rho(x, y)^{\alpha} \wedge\left(c_{3} R^{\alpha}\right)} \frac{t}{\rho(x, y)^{d+\alpha}} d t+\int_{\rho(x, y)^{\alpha} \wedge\left(c_{3} R^{\alpha}\right)}^{c_{3} R^{\alpha}} t^{-d / \alpha} d t\right) \\
& \geqslant c_{6} \rho(x, y)^{-d+\alpha},
\end{aligned}
$$

where in the last inequality we have used the facts that $d>\alpha$ and $\rho(x, y) \leqslant R / 2$ for any $x, y \in B(0, R / 4)$. Combining both estimates above, we obtain (3.12). 
According to Proposition 3.4 and its proof, we can also obtain the following estimates for global Green functions.

Proposition 3.5. Assume that Assumptions (HK1), (HK2) and (HK3) hold with $\theta \in$ $(0,1)$ and $R_{0} \geqslant 1$. We further suppose that $\sup _{r \geqslant 1} \Theta(r)<\infty$. Then, for every $\theta^{\prime} \in(\theta, 1)$, there exists a constant $R_{1} \geqslant 1$ such that for all $x, y \in V$ with

$$
\rho(x, y) \geqslant 4\left(R_{1} \vee \rho(0, x) \vee \rho(0, y)\right)^{\theta^{\prime}},
$$

it holds

$$
C_{1} \rho(x, y)^{-d+\alpha} \leqslant G(x, y) \leqslant C_{2} \rho(x, y)^{-d+\alpha},
$$

where $C_{1}, C_{2}>0$ are independent of $R_{0}, R_{1}, x$ and $y$.

Proof. For any $x, y \in V$, define $D(x, y):=R_{1} \vee \rho(0, x) \vee \rho(0, y)$ with $R_{1} \geqslant 1$ being the constant in Proposition 3.4. Suppose that $x, y \in V$ satisfy $\rho(x, y) \geqslant 4 D(x, y)^{\theta^{\prime}}$. Then, applying (the first inequality in) (3.13) and (3.8), we can follow the proof of Proposition 2.7 with $N(t, x, y):=\rho(x, y)^{(d+\alpha) / \alpha}$ and get that for any $R$ large enough and any $0<t \leqslant$ $\rho(x, y)^{\alpha}$,

$$
p(t, x, y) \leqslant c_{1}\left[\Theta\left(\rho(x, y)^{(d+\alpha) / \alpha}\right)\right]^{k} \frac{t}{\rho(x, y)^{d+\alpha}} \leqslant \frac{c_{2} t}{\rho(x, y)^{d+\alpha}} .
$$

This along with (2.14) yields that

$$
G(x, y) \leqslant c_{3}\left(\int_{0}^{\rho(x, y)^{\alpha}} \frac{t}{\rho(x, y)^{d+\alpha}} d t+\int_{\rho(x, y)^{\alpha}}^{\infty} t^{-d / \alpha} d t\right) \leqslant c_{4} \rho(x, y)^{-d+\alpha},
$$

proving the desired upper bound in (3.14).

On the other hand, the desired lower bound in (3.14) follows from (3.12) by taking $R=4 D(x, y)$. The proof is complete.

3.3. Consequence of elliptic Harnack inequalities. Let $\mathcal{L}$ be the generator associated with the process $X$. For any subset $A \subseteq V$, we say that $u: A \rightarrow \mathbb{R}$ is harmonic with respect to $\mathcal{L}$ on $A$, if for any $x \in A, \mathcal{L} u(x)=0$. We call that the elliptic Harnack inequality (EHI) (associated with $\mathcal{L}$ ) holds at $x_{0} \in V$, if there are constants $R_{0}:=R_{0}\left(x_{0}\right) \geqslant 1$ and $c_{1}:=c_{1}\left(x_{0}, R_{0}\right) \geqslant 1$ such that for every $R \geqslant R_{0}$ and every non-negative function $f(x)$ on $V$ which is harmonic on $B\left(x_{0}, 2 R\right)$, it holds that

$$
\sup _{x \in B\left(x_{0}, R\right)} f(x) \leqslant c_{1}\left(x_{0}, R_{0}\right) \inf _{x \in B\left(x_{0}, R\right)} f(x) .
$$

We emphasize that here we use a weaker version of EHI, where the constant $c_{1}\left(x_{0}, R_{0}\right)$ may depend on $x_{0}$ and $R_{0}$. Note that unlike [29], in the present setting the associated elliptic Harnack inequality is not necessarily translation or scaling invariant.

Proposition 3.6. Let $d>\alpha$. Suppose that Assumptions (HK1), (HK2) and (HK3) hold with some constants $\theta \in(0,1)$ and $R_{0} \geqslant 1$. Assume further that $\sup _{r \geqslant 1} \Theta(r)<\infty$. If EHI holds at $x_{0} \in V$, then for every $\theta^{\prime} \in(\theta, 1)$, there exist constants $R_{1}:=R_{1}\left(x_{0}\right) \geqslant 1$ and $c_{0}:=c_{0}\left(x_{0}, R_{1}\right)>0$ such that for all $R \geqslant R_{1}$ and $z \in B\left(x_{0}, 4 R\right)^{c}$,

$$
w_{x_{0}, z} \leqslant c_{0}\left(\sup _{v \in B\left(x_{0}, 2 R\right), v \neq x_{0}} w_{v, z}\right) R^{\alpha+\theta^{\prime}(d-\alpha)}
$$

Proof. Without loss of generality, we will assume that $x_{0}=0$. For any $R \geqslant 1$ and $z \in B(0,4 R)^{c}$, define

$$
f_{z}(x)=\mathbb{P}_{x}\left(X_{\tau_{B(0,2 R)}}=z\right), \quad x \in B(0,2 R),
$$


which is a harmonic function on $B(0, R)$. By EHI, there are constants $R_{0} \geqslant 1$ and $c_{1}:=$ $c_{1}\left(x_{0}, R_{0}\right) \geqslant 1$ such that for all $z \in B(0,4 R)^{c}$,

$$
f_{z}(x) \leqslant c_{1} f_{z}(y), \quad x, y \in B(0, R) .
$$

Note that, according to the Ikeda-Watanabe formula (see [45, Theorem 2]), it holds that

$$
f_{z}(x)=\sum_{v \in B(0,2 R)} G^{B(0,2 R)}(x, v) \frac{w_{v, z} \mu_{v}}{\rho(v, z)^{d+\alpha}}, \quad x \in B(0, R) .
$$

According to Theorem 2.2 (i.e., [24, Theorem 3.4]), under Assumption (HK1) we have (2.11). In particular, there exists a constant $R_{0} \geqslant 1$ (here for notational simplicity, we take the same $R_{0}$ as above) such that for all $R \geqslant R_{0}$ and $x \in B(0, R)$,

$$
\sum_{y \in B(0,2 R)} G^{B(0,2 R)}(x, y) \mu_{y}=\mathbb{E}_{x}\left[\tau_{B(0,2 R)}\right] \leqslant \mathbb{E}_{x}\left[\tau_{B(x, 4 R)}\right] \leqslant c_{2} R^{\alpha},
$$

where $c_{2}>0$ is independent of $R_{0}$ and $R$. (We note that here $c_{2}$ is also independent of $x_{0}$ if we consider that EHI holds at $x_{0} \in V$ in the beginning.)

Since $\rho(z, 0) / 2 \leqslant \rho(z, v) \leqslant 3 \rho(z, 0) / 2$ for all $z \in B(0,4 R)^{c}$ and $v \in B(0, R)$, for any $R \geqslant R_{0}$ with $R / 2 \geqslant R^{\theta^{\prime}}, y \in B(0, R)$ with $\rho(y, 0) \geqslant R / 2$ and $z \in B(0,4 R)^{c}$,

$$
\begin{aligned}
f_{z}(y)= & \sum_{v \in B(0,2 R)} G^{B(0,2 R)}(y, v) \frac{w_{v, z} \mu_{v}}{\rho(v, z)^{d+\alpha}} \\
\leqslant & c_{3} \rho(z, 0)^{-d-\alpha} \\
& \times\left[\left(\sup _{v \in B(0,2 R), v \neq 0} w_{v, z}\right) \cdot\left(\sum_{v \in B(0,2 R)} G^{B(0,2 R)}(y, v) \mu_{v}\right)+w_{0, z} G^{B(0, R)}(y, 0)\right] \\
\leqslant & c_{4} \rho(z, 0)^{-d-\alpha}\left(R^{\alpha} \cdot \sup _{v \in B(0,2 R), v \neq 0} w_{v, z}+w_{0, z} R^{-d+\alpha}\right),
\end{aligned}
$$

where $c_{4}>0$ is independent of $R_{0}$ and $R$, and in the last inequality we have used (3.17) and (3.11), thanks to the fact that $\rho(y, 0) \geqslant R^{\theta^{\prime}}$.

On the other hand, by (3.12), it holds that

$$
\begin{aligned}
f_{z}(0) & =\sum_{v \in B(0,2 R)} G^{B(0,2 R)}(0, v) \frac{w_{v, z} \mu_{v}}{\rho(v, z)^{d+\alpha}} \\
& \geqslant c_{5} \rho(z, 0)^{-d-\alpha} G^{B(0,2 R)}(0,0) w_{0, z} \geqslant c_{6} \rho(z, 0)^{-d-\alpha} w_{0, z} R^{-\theta^{\prime}(d-\alpha)},
\end{aligned}
$$

where $c_{6}>0$ is independent of $R_{0}$ and $R$.

Combining both estimates above with (3.16), we find that for all $R \geqslant R_{0}$ and $z \in$ $B(0,4 R)^{c}$

$$
w_{0, z} R^{-\theta^{\prime}(d-\alpha)} \leqslant c_{7} w_{0, z} R^{-d+\alpha}+c_{7}\left(\sup _{v \in B(0,2 R), v \neq 0} w_{v, z}\right) R^{\alpha} .
$$

Noting that $c_{7}$ is independent of $R$ (but may depend on $R_{0}$ ) and that $\theta^{\prime}<1$, we can take $R_{1}$ large enough such that $c_{7} R^{-d+\alpha} \leqslant R^{-\theta^{\prime}(d-\alpha)} / 2$ for all $R \geqslant R_{1} \geqslant R_{0}$. Therefore, for all $R \geqslant R_{1}$,

$$
w_{0, z} R^{-\theta^{\prime}(d-\alpha)} \leqslant 2 c_{7}\left(\sup _{v \in B(0,2 R), v \neq 0} w_{v, z}\right) R^{\alpha},
$$

which proves the desired assertion (3.15). 


\section{Application: Random Conductance Model}

We will apply results in the previous two sections to study heat kernel estimates and elliptic Harnack inequalities for random conductance models on $\mathbb{L}:=\mathbb{Z}_{+}^{d_{1}} \times \mathbb{Z}^{d_{2}}$ (with $d_{1}, d_{2} \in \mathbb{Z}_{+}$such that $d_{1}+d_{2} \geqslant 1$ ) with stable-like jumps.

Let $V=\mathbb{L}$ and $\left\{w_{x, y}(\omega): x, y \in \mathbb{L}\right\}$ be a sequence of independent (but not necessarily identically distributed) random variables on some probability space $\left(\Omega, \mathscr{F}_{\Omega}, \mathbb{P}\right)$ such that $w_{x, y}=w_{y, x} \geqslant 0$ for any $x \neq y$, and $w_{x, x}=0$ for any $x \in \mathbb{L}$. Let $\mu$ be a strictly positive (random) measure on $\mathbb{L}$. For $\mathbb{P}$-a.s. fixed $\omega \in \Omega$, we consider the following regular Dirichlet form $\left(D^{\omega}, \mathscr{F}^{\omega}\right)$ on $L^{2}(\mathbb{L} ; \mu)$,

$$
\begin{aligned}
D^{\omega}(f, f) & =\sum_{x, y \in \mathbb{L}}(f(x)-f(y))^{2} \frac{w_{x, y}(\omega)}{|x-y|^{d+\alpha}}, \quad f \in \mathscr{F}^{\omega}, \\
\mathscr{F}^{\omega} & =\left\{f \in L^{2}(\mathbb{L} ; \mu): D^{\omega}(f, f)<\infty\right\} .
\end{aligned}
$$

Note that unlike (1.6) we do not include the term $\mu_{x} \mu_{y}$ in the Dirichlet form above, but it is obviously reduced into (1.6) by replacing $w_{x, y}$ with $\frac{w_{x, y}(\omega)}{\mu_{x} \mu_{y}}$. We prefer to the expression above due to the consistency of notations as those in [24, Section 5.2.1]. Let $\left(X_{t}^{\omega}\right)_{t \geqslant 0}$ be the symmetric Hunt process associated with $\left(D^{\omega}, \mathscr{F} \omega\right)$, whose infinitesimal generator $\mathcal{L}^{\omega}$ is given by

$$
\mathcal{L}^{\omega} f(x):=\frac{1}{\mu_{x}} \sum_{y \in \mathbb{L}}(f(y)-f(x)) \frac{w_{x, y}(\omega)}{|x-y|^{d+\alpha}} .
$$

In the literature, when $\mu$ is the counting measure (resp. $\mu_{x}=\mu_{x}^{\omega}:=\sum_{z \in \mathbb{L}} \frac{w_{x, z}(\omega)}{|x-z|^{d+\alpha}}$ for all $x \in V), X$ is called a variable speed random walk (resp. a constant speed random walk), and denote by $p^{\omega}(t, x, y)$ (resp. $\left.q^{\omega}(t, x, y)\right)$ the heat kernel of the corresponding process $X$.

\subsection{Heat kernel estimates and local limit theorem.}

Theorem 4.1. (Heat kernel estimates for variable speed random walks) Suppose that $d>4-2 \alpha$,

$$
\sup _{x, y \in \mathbb{L}: x \neq y} \mathbb{P}\left(w_{x, y}=0\right)<2^{-4}
$$

and

$$
\sup _{x, y \in \mathbb{L}: x \neq y} \mathbb{E}\left[w_{x, y}^{2 p}\right]<\infty \text { and } \sup _{x, y \in \mathbb{L}: x \neq y} \mathbb{E}\left[w_{x, y}^{-2 q} \mathbb{1}_{\left\{w_{x, y} \neq 0\right\}}\right]<\infty
$$

for $p, q \in \mathbb{Z}_{+}$with

$$
p>\max \left\{\frac{d+1+\theta_{0}}{d \theta_{0}}, \frac{d+1}{2 \theta_{0}(2-\alpha)}\right\} \quad \text { and } q>\frac{d+1+\theta_{0}}{d \theta_{0}},
$$

where $\theta_{0}:=\alpha /(2 d+\alpha)$. Then, for $\mathbb{P}$-a.s. $\omega \in \Omega$ and every $x \in \mathbb{L}$, there is a constant $R_{x}(\omega) \geqslant 1$ such that for all $R>R_{x}(\omega), t>0$ and $y \in \mathbb{L}$ satisfying $t \geqslant\left(|x-y| \vee R_{x}(\omega)\right)^{\theta \alpha}$,

$$
C_{1}\left(t^{-d / \alpha} \wedge \frac{t}{|x-y|^{d+\alpha}}\right) \leqslant p^{\omega}(t, x, y) \leqslant C_{2}\left(t^{-d / \alpha} \wedge \frac{t}{|x-y|^{d+\alpha}}\right),
$$

where $C_{1}, C_{2}>0$ and $\theta \in(0,1)$ are constants independent of $t, x$ and $y$.

Proof. Obviously, the counting measure $\mu$ satisfies Assumptions ( $d$-Vol). Under (4.2), (4.3) and the condition that $\left\{w_{x, y}(\omega): x, y \in \mathbb{L}\right\}$ is a sequence of independent random variables, we can follow the proof of [24, Proposition 5.6] (in particular, the Borel-Cantelli arguments), and prove that there is a constant $\theta \in(0, \alpha /(2 d+\alpha))$ such that for all fixed $x \in \mathbb{L}$ and P-a.s. $\omega \in \Omega$, Assumptions (HK1), (HK2) and (HK3) hold for random conductance $\left\{w_{x, y}(\omega): x, y \in \mathbb{L}\right\}$ with the associated center 0 and constant $R_{0}$ being 
replaced by $x$ and $\widetilde{R}_{x}(\omega) \geqslant 1$ respectively. Therefore, according to Theorem 2.8 and Theorem 2.12 , for every $x \in \mathbb{L}$ and $\mathbb{P}$-a.s. $\omega \in \Omega$, there exists $R_{x}(\omega) \geqslant 1$ such that $(4.4)$ is fulfilled.

Theorem 4.2. (Heat kernel estimates for constant speed random walks) Suppose that $d>4-2 \alpha, w_{x, y}>0$ for all $x \neq y \in \mathbb{L}$,

$$
\inf _{x \in \mathbb{L}} \mu_{x}^{\omega}>0, \quad \sup _{x \in \mathbb{L}} \mu_{x}^{\omega}<\infty
$$

and (4.3) holds with the same constants $p, q \in \mathbb{Z}_{+}$as these in Theorem 4.1. Then for $\mathbb{P}$-a.s. $\omega \in \Omega$ and every $x \in \mathbb{L}$, there is a constant $R_{x}(\omega) \geqslant 1$ such that for all $R>R_{x}(\omega)$ and for all $t>0$ and $y \in \mathbb{L}$ satisfying $t \geqslant\left(|x-y| \vee R_{x}(\omega)\right)^{\theta_{1} \alpha}$,

$$
C_{3}\left(t^{-d / \alpha} \wedge \frac{t}{|x-y|^{d+\alpha}}\right) \leqslant q^{\omega}(t, x, y) \leqslant C_{4}\left(t^{-d / \alpha} \wedge \frac{t}{|x-y|^{d+\alpha}}\right),
$$

where $C_{3}, C_{4}>0$ and $\theta \in(0,1)$ are constants independent of $t, x$ and $y$.

Proof. For the constant speed random walk, $\mu_{x}^{\omega}=\sum_{z \in \mathbb{L}} \frac{w_{x, z}(\omega)}{|x-z|^{d+\alpha}}$. Then, the associated Dirichlet form enjoys the expression (1.6) with $\mu_{x}=\mu_{x}^{\omega}$ and $w_{x, y}=\frac{w_{x, y}(\omega)}{\mu_{x}^{\omega} \mu_{y}^{\omega}}$. Under (4.5), there are constants $0<c_{1} \leqslant c_{2}<\infty$ such that for all $x \in \mathbb{L}, c_{1} \leqslant \mu_{x}^{\omega} \leqslant c_{2}$, which implies that Assumption ( $d$-Vol) holds. Due to the fact that $\mu_{x}^{\omega}$ is uniformly bounded from upper and below, we can follow in the proof of Theorem 4.1 to verify that for all fixed $x \in \mathbb{L}$ and P-a.s. $\omega \in \Omega$, Assumptions (HK1), (HK2) and (HK3) hold with the associated center $x$ and constant $\widetilde{R}_{x}(\omega) \geqslant 1$ respectively. Therefore, the desired assertion follows from Theorem 2.8 and Theorem 2.12.

Remark 4.3. (1) Similar to the case for variable speed random walks (see Theorem 4.1), we can allow the conductance in Theorem 4.2 to be degenerate; that is, Theorem 4.2 still holds, even if the assumption that $w_{x, y}>0$ for all $x, y \in \mathbb{L}$ is replaced by an upper bound of the probability for degenerate conductances like (4.2). As seen from the proof of [24, Proposition 5.6], such an explicit upper bound depends on the constants $c_{1}, c_{2}$ for uniform bounds of $\left\{\mu_{x}^{\omega}\right\}_{x \in \mathbb{L}}$. We omit the details here.

(2) For the nearest neighbor random conductance models (see e.g. [1, 11, 51]), percolation estimates are crucially used to remove or weaken the condition (4.5). However, such estimates are not available (and are not easy to gain at least) for our model. This explains the reason why we need assume (4.5) in Theorem 4.2.

According to [34, Theorem 1] (see [13, Section 4] or [3, Theorem 1.11] for related discussions), we have the following local limit theorem for $\left(X_{t}^{\omega}\right)_{t \geqslant 0}$. For any $a>0$, let $k_{a, t}(x):=k_{a, t}(0, x)$ be the transition density function corresponding to symmetric $\alpha$-stable processes with Lévy measure $a|z|^{-d-\alpha} d z$.

Theorem 4.4 (Local limit theorem for variable speed random walks). Under the setting of Theorem 4.1, assume further that $\mathbb{E} w_{x, y}=a$ for all $x \neq y \in \mathbb{L}$. Then, for any $T_{2}>T_{1}>0$ and $k>1$,

$$
\lim _{n \rightarrow \infty} \sup _{|x| \leqslant k} \sup _{t \in\left[T_{1}, T_{2}\right]}\left|n^{d} p^{\omega}\left(n^{\alpha} t, 0,[n x]\right)-k_{a, t}(x)\right|=0,
$$

where $[x]=\left(\left[x_{1}\right], \cdots,\left[x_{d}\right]\right)$ for any $x=\left(x_{1}, \cdots, x_{d}\right) \in \mathbb{R}_{+}^{d_{1}} \times \mathbb{R}^{d_{2}}$.

Proof. We consider the scaling limit procedure as used in [24, Section 4], and adopt the notations in [34]. Let $E=F=\mathbb{R}_{+}^{d_{1}} \times \mathbb{R}^{d_{2}}, G=\mathbb{L}$ with $d(x, y)=|x-y|$, and $G^{n}=$ $n^{-1} \mathbb{L}$ with $d_{G^{n}}(x, y)=n d(x, y)$. Denote by $\nu$ the Lebesgue measure, and by $\nu^{n}$ the counting measure on $G^{n}$. It is clear that conditions (a)-(c) in [34, Assumption 1] hold with $\alpha(n)=n, \beta(n)=n^{d}$ and $\gamma(n)=n^{\alpha}$. Let $X_{t}^{n, \omega}:=n^{-1} X_{n^{\alpha} t}^{\omega}$ for any $t>0$, and denote 
by $p^{n, \omega}(t, x, y)$ its heat kernel on $G^{n}=n^{-1} \mathbb{L}$ with respect to the counting measure $\nu^{n}$. Then, $p^{n, \omega}(t, x, y)=p^{\omega}\left(n^{\alpha} t, n x, n y\right)$ for all $x, y \in n^{-1} \mathbb{L}$ and $t>0$, and so it suffices to prove that the conclusion of [34, Theorem 1] holds for $p^{n, \omega}(t, x, y)$. Note that, though [34, Theorem 1] is stated for the local limit theorem for a discrete time Markov process, by carefully tracking the argument, we can verify that the proof also works for a continuous time Markov process, see the proof of [3, Theorem 1.11] for more details. Now, we are going to check that (d) in [34, Assumption 1] holds for $p^{n, \omega}(t, x, y)$.

According to [24, Theorem 1.1], under the setting of Theorem 4.1, the quenched invariance principle holds for $\left(X_{t}^{\omega}\right)_{t \geqslant 0}$ with the limit process being a (reflected) symmetric $\alpha$-stable Lévy process on $\mathbb{R}_{+}^{d_{1}} \times \mathbb{R}^{d_{2}}$ with jumping measure $a|z|^{-d-\alpha} d z$. In particular, this implies that for every $f \in C_{b}\left(\mathbb{R}_{+}^{d_{1}} \times \mathbb{R}^{d_{2}}\right), t>0$ and $x \in \mathbb{R}_{+}^{d_{1}} \times \mathbb{R}^{d_{2}}$,

$$
\lim _{n \rightarrow \infty}\left|\mathbf{E}_{[x]_{n}}^{n, \omega}\left[f\left(X_{t}^{n, \omega}\right)\right]-\int_{\mathbb{R}^{d}} f(z) k_{a, t}(x-z) d z\right|=0,
$$

where $[x]_{n}:=n^{-1}[x]$ and $\mathbf{E}_{x}^{n, \omega}$ denotes the expectation with respect to the law of process $\left(X_{t}^{n, \omega}\right)_{t \geqslant 0}$ with the initial point $x \in n^{-1} \mathbb{L}$. Combining this with Theorem 2.10 (which was used to estimate the term $\left|\mathbf{E}_{[x]_{n}}^{n, \omega}\left[f\left(X_{t}^{n, \omega}\right)\right]-\mathbf{E}_{[x]_{n}}^{n, \omega}\left[f\left(X_{s}^{n, \omega}\right)\right]\right|$ for $0<s<t$ ), we can further verify that for every $f \in C_{b}\left(\mathbb{R}_{+}^{d_{1}} \times \mathbb{R}^{d_{2}}\right), 0<T_{1}<T_{2}$ and $x \in \mathbb{R}_{+}^{d_{1}} \times \mathbb{R}^{d_{2}}$,

$$
\lim _{n \rightarrow \infty} \sup _{t \in\left[T_{1}, T_{2}\right]}\left|\mathbf{E}_{[x]_{n}}^{n, \omega}\left[f\left(X_{t}^{n, \omega}\right)\right]-\int_{\mathbb{R}^{d}} f(z) k_{a, t}(x-z) d z\right|=0
$$

Since for every $x_{0} \in \mathbb{R}_{+}^{d_{1}} \times \mathbb{R}^{d_{2}}$ and $r>0, \int_{\partial B\left(x_{0}, r\right)} k_{a, t}(x-z) d z=0,(4.7)$ in turn yields that

$$
\lim _{n \rightarrow \infty} \sup _{t \in\left[T_{1}, T_{2}\right]}\left|\mathbf{P}_{[x]_{n}}^{n, \omega}\left(X_{t}^{n, \omega} \in B\left(x_{0}, r\right)\right)-\int_{B\left(x_{0}, r\right)} f(z) k_{a, t}(x-z) d z\right|=0,
$$

see e.g. [18, Theorem 2.1]. Therefore, (d) in [34, Assumption 1] is satisfied.

On the other hand, it follows from Theorem 2.10 again and the on-diagonal upper bound (see (4.4)) of $p^{\omega}(t, x, y)$, there are constants $\delta \in(0,1)$ and $\beta \in(0,1)$ such that for any $0<T_{1}<T_{2}, r>0, \gamma \in(0, r]$ and $n \geqslant 1$ large enough,

$$
\sup _{\substack{x, y \in B_{G} n(0, \alpha(n) r), t \in\left[T_{1}, T_{2}\right] \\(n \gamma)^{\delta} \leqslant d_{G_{n}}(x, y) \leqslant n \gamma}} \sup \left|p^{\omega}\left(n^{\alpha} t, 0,[n x]\right)-p^{\omega}\left(n^{\alpha} t, 0,[n y]\right)\right| \leqslant c_{1}\left(T_{1}, T_{2}, r\right) n^{-d} \gamma^{\beta},
$$

where $c_{1}>0$ is independent of $n$ and $\gamma$, and $B_{G^{n}}(0, r)$ is denoted by the ball on $G^{n}$ with center 0 and radius $r$. Note that, (4.8) is slightly weaker than [34, Assumption 2], since we require to take the supremum of $(n \gamma)^{\delta} \leqslant d_{G_{n}}(x, y)$. However, according to (4.8) and the on-diagonal upper bound of heat kernel (4.4), we can get that for every $x \in \mathbb{R}_{+}^{d_{1}} \times \mathbb{R}^{d_{2}}$, $t \in\left[T_{1}, T_{2}\right]$, each fixed $\delta>0$ and $r \in(0, \delta]$ small enough,

$$
\begin{aligned}
\left|J_{1}(t, n, x, r)\right|:= & n^{-d}\left|\sum_{y \in G^{n}: y \in B(x, r)}\left(p^{n, \omega}(t, 0, y)-p^{n, \omega}(t, 0, x)\right)\right| \\
\leqslant & n^{-d}\left|\sum_{y \in G^{n}:(n r)^{\delta} \leqslant d_{G_{n}}(y, x) \leqslant n r}\left(p^{n, \omega}(t, 0, y)-p^{n, \omega}(t, 0, x)\right)\right| \\
& +n^{-d}\left|\sum_{y \in G^{n}: d_{G_{n}}(y, x) \leqslant(n r)^{\delta}}\left(p^{n, \omega}(t, 0, y)-p^{n, \omega}(t, 0, x)\right)\right| \\
\leqslant & n^{-d} \sup _{(n r)^{\delta} \leqslant d_{G_{n}}(y, x) \leqslant n r}\left|p^{n, \omega}(t, 0, y)-p^{n, \omega}(t, 0, x)\right| \cdot \nu^{n}(B(x, r))
\end{aligned}
$$




$$
\begin{aligned}
& +2 n^{-d} \sup _{d_{G_{n}}(y, x) \leqslant(n r)^{\delta}}\left|p^{n, \omega}(t, 0, y)\right| \cdot \nu^{n}\left(B\left(x, n^{-1}(n r)^{\delta}\right)\right) \\
\leqslant & c_{2}\left(T_{1}, T_{2}, \delta\right) n^{-d}\left[\nu^{n}(B(x, r)) n^{-d} r^{\beta}+n^{-d} \nu^{n}\left(B\left(x, n^{-1+\delta} r^{\delta}\right)\right)\right] \\
\leqslant & c_{3}\left(T_{1}, T_{2}, \delta\right) n^{-d}\left[r^{d+\beta}+n^{-(1-\delta) d} r^{\delta d}\right] .
\end{aligned}
$$

Hence

$$
\lim _{r \rightarrow 0} \limsup _{n \rightarrow \infty} \sup _{t \in\left[T_{1}, T_{2}\right]} n^{d}\left|J_{1}(t, n, x, r)\right|=0 .
$$

With this estimate replacing that of $J_{1}(t, n)$ in the proof of [13, Theorem 4.2], the proof of [34, Theorem 1] is valid. See also the proof of [3, Theorem 1.11].

Therefore, the desired assertion follows from [34, Theorem 1].

Remark 4.5. Here we take $V=\mathbb{L}$ in Theorem 4.1 and Theorem 4.4 for simplicity. As in $[24$, Section 5], these results also hold for more general $d$-sets, including the Sierpinski gasket.

\subsection{Two counterexamples.}

4.2.1. Heat kernel estimates. In this subsection, we give an example that shows the heat kernel may behave anomalously without the moment condition of $w_{x y}^{-1}$. This example is heavily motivated by [17, Theorem $2.1(1)]$.

In the following, denote by $P^{\omega}(n, x, y)$ the $n$-step transition probability for discrete time Markov chain associated with the conductance $\left\{C_{x, y}(\omega):=\frac{w_{x, y}(\omega)}{\left.|x-y|\right|^{d+\alpha}}: x, y \in \mathbb{Z}^{d}\right\}$, and by $q^{\omega}(t, x, y)$ the heat kernel for the constant speed random walk associated with $\left\{C_{x, y}(\omega): x, y \in \mathbb{Z}^{d}\right\}$.

Proposition 4.6. Let $d \geqslant 5$ and $\kappa>1 / d$. Then, there exist a sequence of independent random variables $\left\{w_{x, y}: x, y \in \mathbb{Z}^{d}\right\}$ such that $w_{x, y} \stackrel{d}{=} w_{x^{\prime}, y^{\prime}}$ for $|x-y|=\left|x^{\prime}-y^{\prime}\right|$, and

$$
\mathbb{P}\left(w_{x, y} \leqslant 1\right)=1, c_{1}^{-1} \leqslant \mathbb{E}\left[w_{x, y}\right] \leqslant c_{1}, \quad \forall x \neq y \in \mathbb{Z}^{d},
$$

for some constant $c_{1} \geqslant 1$ independent of $x$ and $y$; while

$$
P^{\omega}(2 n, 0,0) \geqslant C(\omega) \frac{e^{-(\log n)^{\kappa}}}{n^{2}}, \quad \forall n \geqslant 1, \text { a.s. } \mathbb{P}
$$

for some random variable $C(\omega)>0$. In particular, for any $\delta>0$ small enough, there exists a constant $C_{1}(\omega)>0$ such that

$$
q^{\omega}(t, 0,0) \geqslant C_{1}(\omega) t^{-2-\delta}, \quad t \geqslant 1 .
$$

Proof. Since

$$
q^{\omega}(t, x, y)=\sum_{n \geqslant 0} \frac{t^{n}}{n !} e^{-t} P^{\omega}(n, x, y), \quad t>0, x, y \in \mathbb{Z}^{d},
$$

it suffices to prove (4.10). The proof is similar to that of [17, Theorem 2.1(1)] except that we should control long range bonds suitably. For $\kappa>1 / d$, choose $\varepsilon>0$ small enough so that $(1+(4 d+2) \varepsilon) / d<\kappa$. Take a sequence of independent random variables $\left\{w_{x, y}: x, y \in \mathbb{Z}^{d}\right\}$ such that

(i) for every $|x-y|=1$, we set

$$
\mathbb{P}\left(w_{x, y}=1\right)>p_{c}(d), \mathbb{P}\left(w_{x, y}=2^{-N}\right)=c_{1} N^{-(1+\varepsilon)}, \forall N \geqslant 1,
$$

for some $c_{1}>0$, where $p_{c}(d)$ is the critical probability of the Bernoulli percolation on $\mathbb{Z}^{d}$; 
(ii) for $|x-y|>1$, choose $\left\{w_{x, y}: x, y \in \mathbb{Z}^{d}\right\}$ to satisfy (4.9) and

$$
\mathbb{P}\left(\sum_{z \in \mathbb{L}:|y-z|>1} C_{y, z} \leqslant M\right)=1, \mathbb{P}\left(\sum_{z \in \mathbb{L}:|y-z|>1} C_{y, z} \leqslant 2^{-N}\right) \geqslant c_{2} N^{-\varepsilon}, \forall N \geqslant 1,
$$

for some $M \geqslant 1$ and $c_{2}>0$, where $C_{x, y}=w_{x, y} /|x-y|^{d+\alpha}$.

Define

$$
\ell_{N}=N^{(1+(4 d+2) \varepsilon) / d}
$$

and for each $x \in \mathbb{Z}^{d}$, let $A_{N}(x)$ be the event that the configuration near $y=x+\mathbf{e}_{1}$ and $z=x+2 \mathbf{e}_{1}$ (here $\mathbf{e}_{1}=(1,0, \cdots, 0)$ ) is as follows:

(iii) $C_{y, z}=1, C_{x, y}=2^{-N}$ and other nearest neighbor bonds (that is, bonds with length 1 ; n.n. bonds in short) connected to $y$ and $z$ have conductance $\leqslant 2^{-N}$; moreover, $\sum_{u \in \mathbb{L}:|y-u|>1} C_{y, u} \leqslant 2^{-N}$ and $\sum_{u \in \mathbb{L}:|z-u|>1} C_{z, u} \leqslant 2^{-N}$.

(iv) $x$ is connected to the boundary of the box of side length $\left(\log \ell_{N}\right)^{2}$ centered at $x$ by n.n. bonds and conductance 1 .

Since bonds with conductance 1 percolate, using (4.11) and (4.12), we have

$$
\mathbb{P}\left(A_{N}(x)\right) \geqslant c_{3} N^{-1-\varepsilon} \cdot N^{-(4 d-3) \varepsilon} \cdot N^{-2 \varepsilon}=c_{3} N^{-(1+4 d \varepsilon)},
$$

where we used the fact that $\mathbb{P}\left(w_{x, y} \leqslant 2^{-N}\right) \leqslant c_{*} N^{-\varepsilon}$ for all $x, y \in \mathbb{Z}^{d}$ with $|x-y|=1$. Now, let $\mathbb{G}_{n}$ be a grid of vertices in $\left[-\ell_{N}, \ell_{N}\right]^{d} \cap \mathbb{Z}^{d}$ that are located by distance $2\left(\log \ell_{N}\right)^{2}$. Then, the events $\left\{A_{N}(x): x \in \mathbb{Z}^{d}\right\}$ are independent, and

$$
\mathbb{P}\left(\bigcap_{x \in \mathbb{G}_{n}} A_{N}(x)^{c}\right) \leqslant \exp \left(-c_{4}\left(\frac{\ell_{N}}{\left(\log \ell_{N}\right)^{2}}\right)^{d} N^{-(1+4 d \varepsilon)}\right) \leqslant e^{-c_{5} N^{\varepsilon}},
$$

hence the intersection occurs only for finitely many $N$.

Given this stretched-exponential decay, we know that every connected component of length $\left(\log \ell_{N}\right)^{2}$ in $\left[-\ell_{N}, \ell_{N}\right]^{d} \cap \mathbb{Z}^{d}$ is connected to the largest connected component in $\left[-2 \ell_{N}, 2 \ell_{N}\right]^{d} \cap \mathbb{Z}^{d}$ by using only n.n. bonds for large enough $N$ (see [44, Theorem 8.65]). Hence, there exists $N_{0}=N_{0}(\omega)<\infty$ such that for any $N \geqslant N_{0}, A_{N}(x)$ occurs for some even-parity vertex $x=x_{N}(\omega) \in\left[-\ell_{N}, \ell_{N}\right]^{d} \cap \mathbb{Z}^{d}$ which is connected to 0 by a path (say $\operatorname{Path}_{N}$ ) in $\left[-2 \ell_{N}, 2 \ell_{N}\right]^{d} \cap \mathbb{Z}^{d}$, on which only the $N_{0}$ n.n. bonds close to the origin may have conductance smaller than 1 .

Now suppose $N \geqslant N_{0}$ and let $n$ be such that $2^{N} \leqslant 2 n<2^{N+1}$. Let $x_{N}=x_{N}(\omega)$ be as above and $r_{N}$ be the length of $\operatorname{Path}_{N}$. Let $\alpha=\alpha(\omega)$ be the minimum of the conductance in the n.n. bonds within $N_{0}$ steps from the origin. Then the passage from the origin to $x_{N}$ in time $r_{N}$ has probability $\geqslant \alpha^{N_{0}} C_{*}^{-r_{N}}$ where $C_{*}=\sum_{|y| \geqslant 1}|y|^{-d-\alpha}>2 d$ (due to the fact that $w_{x, y} \leqslant 1$ for all $\left.x, y \in \mathbb{Z}^{d}\right)$, while the probability of staying on the bond $(y, z)$ for time $2 n-2 r_{N}-2$ is bounded from below by a constant which is independent of $\omega$. The transition probability across $(x, y)$ is of order $2^{-N}$. Hence, we have

$$
P^{\omega}(2 n, 0,0) \geqslant c_{6} \alpha^{2 N_{0}} C_{*}^{-2 r_{N}} 2^{-2 N} .
$$

Using the comparison of the graph distance and the Euclidean distance (see [7]), we have $r_{N} \leqslant c \ell_{N}$ for $N$ large enough. Since $n \asymp 2^{N}$, we obtain the desired estimate.

Finally, let us give a concrete example that satisfies (4.12). The first equality in (4.12) is an easy consequence of (4.9). Now, for any $l \geqslant 1$, define $f_{l}(s)=\left(\log _{2}\left(c_{0} / s\right)\right)^{-\varepsilon^{\prime} l^{-1-d}}$ for $s \leqslant 1 / 2$ small enough ( $\varepsilon^{\prime}$ is a small positive value chosen later). For any $y, z \in \mathbb{Z}^{d}$ with $|y-z|=l$, let $\mathbb{P}\left(\omega_{y, z}=1\right) \geqslant 1 / 2$ and $\mathbb{P}\left(\omega_{y, z} \leqslant s\right)=f_{l}(s)$ (by possibly choosing the 
constant $c_{0}$ and the range of $s$ in the definition of $\left.f_{l}(s)\right)$. Then, we have

$$
\begin{aligned}
\mathbb{P}\left(\sum_{|y-z|>1} C_{y, z} \leqslant 2^{-N}\right) & \geqslant \mathbb{P}\left(\bigcap_{l=2}^{\infty} \bigcap_{|y-z|=l}\left\{w_{y, z} \leqslant c^{\prime} 2^{-N}\right\}\right) \geqslant \prod_{l=2}^{\infty} f_{l}\left(c^{\prime} 2^{-N}\right)^{c l^{d-1}} \\
& \geqslant c^{\prime \prime} \prod_{l=2}^{\infty} N^{-\varepsilon^{\prime} l^{-1-d} \cdot c l^{d-1}}=c^{\prime \prime} N^{-c \varepsilon^{\prime} \sum_{l=2}^{\infty} l^{-2}}=c^{\prime \prime} N^{-c^{\prime \prime \prime} \varepsilon^{\prime}} .
\end{aligned}
$$

Choosing $\varepsilon^{\prime}=\varepsilon / c^{\prime \prime \prime}$, we obtain the inequality in (4.12).

4.2.2. Elliptic Harnack inequalities. Before presenting a counterexample such that EHI does not hold for random conductance models with non-uniformly elliptic stable-like jumps, we give the following statement, which is a consequence of Proposition 3.6.

Proposition 4.7. Under the setting of Theorem 4.1 with $d_{1}=0$ (i.e. $\mathbb{L}=\mathbb{Z}^{d}$ ) and $d>\alpha$, assume further that $\left\{w_{x, y}: x, y \in \mathbb{Z}^{d}\right\}$ is a sequence of independent random variables so that

$$
\begin{gathered}
\sup _{x, y \in \mathbb{Z}^{d}: x \neq y} w_{x, y}^{-1}<\infty, \quad \text { a.s. } \mathbb{P}, \\
\inf _{x, y \in \mathbb{Z}^{d}: x \neq y} \mathbb{P}\left(w_{x, y} \leqslant c_{0}\right)>0 \quad \text { for some } c_{0}>0,
\end{gathered}
$$

and

$$
\inf _{x, y \in \mathbb{Z}^{d}: x \neq y} \mathbb{P}\left(w_{x, y}>k\right)>0 \quad \text { for any } k>0 .
$$

Then, for $\mathbb{P}$-a.s. $\omega \in \Omega$, EHI does not hold at any $x_{0} \in \mathbb{Z}^{d}$.

Proof. Without loss of generality, we only verify the case that $x_{0}=0$. As explained in the proof of Theorem 4.1, for P-a.s. $\omega \in \Omega$, Assumptions (HK1), (HK2) and (HK3) hold with some constant $\theta^{\prime} \in(0, \alpha /(2 d+\alpha))$. According to Proposition 3.6, it suffices to disprove the inequality (3.15).

For every fixed $R \geqslant 1, N>0$ and $z \in B(0,4 R)^{c}$, we set

$$
p(R, N, z):=\mathbb{P}\left(w_{0, z} \leqslant N R^{\alpha+\theta^{\prime}(d-\alpha)} \sup _{v \in B(0,2 R), v \neq 0} w_{v, z}\right) .
$$

Note that here $\theta^{\prime} \in(0, \alpha /(2 d+\alpha))$ is independent of $R$ and $N$.

Since $\left\{w_{x, y}: x, y \in \mathbb{Z}^{d}\right\}$ is a sequence of independent random variables, for the constant $c_{0}$ given in (4.14) it holds that

$$
\begin{aligned}
p(R, N, z) & =1-\prod_{v \in B(0,2 R), v \neq 0} \mathbb{P}\left(w_{0, z}>N R^{\alpha+\theta^{\prime}(d-\alpha)} w_{v, z}\right) \\
& \leqslant 1-\prod_{v \in B(0,2 R), v \neq 0} \mathbb{P}\left(w_{0, z}>N R^{\alpha+\theta^{\prime}(d-\alpha)} c_{0}, w_{v, z} \leqslant c_{0}\right) \\
& =1-\prod_{v \in B(0,2 R), v \neq 0} \mathbb{P}\left(w_{0, z}>N R^{\alpha+\theta^{\prime}(d-\alpha)} c_{0}\right) \mathbb{P}\left(w_{v, z} \leqslant c_{0}\right) \\
& \leqslant 1-c_{1}(R, N)^{R^{d}}:=c_{2}(R, N) .
\end{aligned}
$$

Here, by (4.14) and (4.15), $c_{1}(R, N)$ and $c_{2}(R, N)$ are positive constants which can be chosen to be independent of $z$. Therefore, for every fixed $N$ and $R$, and any $k_{0} \geqslant 1$,

$$
\sum_{k=k_{0}}^{\infty} \mathbb{P}\left(\bigcap_{z \in \mathbb{Z}^{d: 2^{k}<|z| \leqslant 2^{k+1}}}\left\{w_{0, z} \leqslant N R^{\alpha+\theta^{\prime}(d-\alpha)} \sup _{v \in B(0,2 R), v \neq 0} w_{v, z}\right\}\right)
$$




$$
\leqslant \sum_{k=k_{0}}^{\infty} \prod_{z \in \mathbb{Z}^{d}: 2^{k}<|z| \leqslant 2^{k+1}} p(R, N, z) \leqslant \sum_{k=k_{0}}^{\infty} c_{2}(R, N)^{2^{k}}<\infty .
$$

This along with the Borel-Cantelli lemma yields that for every $R \geqslant 1, N>0$ and $\mathbb{P}$-a.s. $\omega \in \Omega$, there exists a constant $k_{1}:=k_{1}(\omega, R, N)>0$ such that for all $k \geqslant k_{1}$, we can find $z \in \mathbb{Z}^{d}$ with $2^{k}<|z| \leqslant 2^{k+1}$ satisfying that

$$
w_{0, z}>N R^{\alpha+\theta^{\prime}(d-\alpha)} \sup _{v \in B(0,2 R), v \neq 0} w_{v, z} .
$$

In particular, (3.15) does not hold true. The proof is finished.

Example 4.8 (Counterexample for EHI). Let $\left\{w_{x, y}: x, y \in \mathbb{Z}^{d}\right\}$ be a sequence of i.i.d. random variables on some probability space $(\Omega, \mathscr{F}, \mathbb{P})$ such that for $w_{x, y}=w_{y, x}>0$ for any $x \neq y$ and $w_{x, x}=0$ for any $x \in \mathbb{Z}^{d}$. Suppose that $d>\alpha$. Let $p>0$ be that constant given in Theorem 4.1, and let $\varepsilon>0$. We assume that for any $x \neq y \in \mathbb{Z}^{d}$

$$
\mathbb{P}\left(w_{x, y}=k\right)=k^{-(2 p+1+\varepsilon)}, \quad k \in \mathbb{Z}_{+} \text {with } k \geqslant 2,
$$

and that

$$
\mathbb{P}\left(w_{x, y}=1\right)=1-\sum_{k=2}^{\infty} \mathbb{P}\left(w_{x, y}=k\right) .
$$

Then, assumptions in Theorem 4.1 and Proposition 4.7 are fulfilled. Therefore, according to Proposition 4.7, with this choice of $\left\{w_{x, y}\right\}$ the EHI does not hold for any $x_{0} \in \mathbb{Z}^{d}$.

Remark 4.9. Concerning nearest neighbor models, the equivalence between heat kernel estimates and parabolic Harnack inequalities, both of which only hold for sufficiently large balls, was established in [10, Theorem 1.10]. However, the proof (see that of [10, Theorem 7.2]) crucially uses some priori estimates of heat kernel for small time, see [10, (3.9)]. As we mentioned above, such estimates are not available in the present setting.

We close this section with one remark on weak elliptic Harnack inequalities (WEHI) for random conductance models with stable-like jumps. The reader can refer to [29] and references therein for more details on WEHI for non-local Dirichlet forms.

Remark 4.10. Under the setting of Theorem 4.1 with $d_{1}=0$ (i.e. $\mathbb{L}=\mathbb{Z}^{d}$ ) and $d>\alpha$, if (4.13) holds, then for $\mathbb{P}$-a.s. $\omega \in \Omega$ and every $x \in \mathbb{Z}^{d}$, there exists a constant $R_{x}(\omega) \geqslant 1$ such that for any $R>R_{x}(\omega)$ and positive harmonic function $h$ on $B(x, 2 R)$, it holds that

$$
\frac{1}{R^{d}} \sum_{z \in B(x, R)} h(z) \leqslant c_{0} \inf _{z \in B(x, R)} h(z)
$$

where $c_{0}>0$ is a non-random constant independent of $x, R_{x}(\omega), R$ and $h$. Note that, by Proposition 4.7, EHI does not hold under (4.13)-(4.15), and so Example 4.8 provides us a concrete example that WEHI holds but EHI fails for random conductance models with stable-like jumps.

The conclusion (4.16) mainly follows from Moser's iteration procedures as in the proof of [39, Theorem 1.1] (see also the proof of [38, Theorem 4.1]), which are based on the following three ingredients.

First, under (4.13), it is a direct consequence of the discrete fractional Sobolev inequality on $\mathbb{Z}^{d}$ (with $d>\alpha$ ) that for all $R \geqslant 1$ and $x \in \mathbb{Z}^{d}$,

$$
\begin{gathered}
\left(\sum_{z \in B(x, R)} f^{2 d /(d-\alpha)}(z)\right)^{(d-\alpha) / d} \leqslant \\
c_{1} \sum_{y, z \in B(x, R)}(f(x)-f(y))^{2} \frac{w_{y, z}(\omega)}{|y-z|^{d+\alpha}} \\
+c_{1} R^{-\alpha} \sum_{z \in B(x, R)} f^{2}(z)
\end{gathered}
$$


where $c_{1}>0$ is independent of $x, R$ and $f$. Second, also due to (4.13), we can follow the arguments in [37, Corollary 5] and use the scaling property to establish the following weighted Poincaré inequality

$$
\begin{aligned}
& \sum_{z \in B(x, 3 R / 2)}\left(f(z)-f_{\Psi_{R}}\right)^{2} \Psi_{R}(z) \\
\leqslant & c_{2} R^{\alpha} \sum_{y, z \in B(x, 3 R / 2)}(f(x)-f(y))^{2} \frac{w_{y, z}(\omega)}{|y-z|^{d+\alpha}} \Psi_{R}(y) \wedge \Psi_{R}(z),
\end{aligned}
$$

where $\Psi_{R}(x):=((3 R / 2)-|x|) \wedge R$,

$$
f_{\Psi_{R}}:=\left(\sum_{z \in B(x, 3 R / 2)} \Psi_{R}(z)\right)^{-1}\left(\sum_{z \in B(x, 3 R / 2)} f(z) \Psi_{R}(z)\right)
$$

and $c_{2}$ is independent of $x, R$ and $f$. Third, as explained in the proof of Theorem 4.1, under (4.3), we know that for $\mathbb{P}$-a.s. $\omega \in \Omega$ and every $x \in \mathbb{Z}^{d}$, there exists a constant $R_{x}(\omega) \geqslant 1$ such that for all $R>R_{x}(\omega)$ and $R^{\theta} \leqslant r \leqslant 2 R$,

$$
\sup _{y \in(0,2 R)} \sum_{z \in \mathbb{Z}^{d}:|y-z| \leqslant r} \frac{w_{x, y}(\omega)}{|z-y|^{d+\alpha-2}} \leqslant c_{3} r^{-\alpha}
$$

and

$$
\sup _{y \in(0,2 R)} \sum_{z \in \mathbb{Z}^{d}:|y-z|>r} \frac{w_{x, y}(\omega)}{|z-y|^{d+\alpha}} \leqslant c_{3} r^{-\alpha},
$$

where $\theta \in(0,1)$ are $c_{3}>0$ are independent of $x \in \mathbb{Z}^{d}, R_{x}(\omega), r$ and $R$.

We finally emphasize that the argument above is based on the condition (4.13). It is an interesting question to prove fractional Sobolev inequalities and weighted Poincaré inequalities as above under weaker condition than (4.13).

Acknowledgements. The research of Xin Chen is supported by the National Natural Science Foundation of China (No. 11501361). The research of Takashi Kumagai is supported by JSPS KAKENHI Grant Number JP17H01093 and by the Alexander von Humboldt Foundation. The research of Jian Wang is supported by the National Natural Science Foundation of China (No. 11522106), the Fok Ying Tung Education Foundation (No. 151002), and the Program for Probability and Statistics: Theory and Application (No. IRTL1704).

\section{REFERENCES}

[1] Andres, S., Barlow, M.T., Deuschel, J.-D. and Hambly, B. M.: Invariance principle for the random conductance model, Probab. Theory Related Fields 156 (2013), 535-580.

[2] Andres, S., Deuschel, J.-D. and Slowik, M.: Invariance principle for the random conductance model in a degenerate ergodic environment, Ann. Probab. 43 (2015), 1866-1891.

[3] Andres, S., Deuschel, J.-D. and Slowik, M.: Harnack inequalities on weighted graphs and some applications to the random conductance model, Probab. Theory Related Fields 164 (2016), 931-977.

[4] Andres, S., Deuschel, J.-D. and Slowik, M.: Heat kernel estimates for random walks with degenerate weights, Electron. J. Probab 21 (2016), paper no. 33, 21 pp.

[5] Andres, S., Deuschel, J.-D. and Slowik, M.: Harnack inequalities on weighted graphs and some applications to the random conductance model, Probab. Theory Relat. Fields 166 (2017), 713-752.

[6] Andres, S., Chiarini, A., Deuschel, J.-D. and Slowik, M.: Quenched invariance principle for random walks with time-dependent ergodic degenerate weights, Ann. Probab. 46 (2018), 302-336.

[7] Antal, P. and Pisztora, A.: On the chemical distance for supercritical Bernoulli percolation. Ann. Probab. 24 (1996), 1036-1048.

[8] Barlow, M.T.: Random walks on supercritical percolation clusters, Ann. Probab. 32 (2004), 30243084.

[9] Barlow, M.T., Bass, R.F. and Kumagai, T.: Parabolic Harnack inequality and heat kernel estimates for random walks with long range jumps, Math. Z. 261 (2009), 297-320. 
[10] Barlow, M.T. and Chen, X.X.: Gaussian bounds and parabolic Harnack inequality on locally irregular graphs, Math. Ann. 366 (2016), 1677-1720.

[11] Barlow, M.T. and Deuschel, J.-D.: Invariance principle for the random conductance model with unbounded conductances, Ann. Probab. 38 (2010), 234-276.

[12] Barlow, M.T., Grigor'yan, A. and Kumagai, T.: Heat kernel upper bounds for jump processes and the first exit time, J. Reine Angew. Math. 626 (2009), 135-157.

[13] Barlow, M.T. and Hambly, B.M.: Parabolic Harnack inequality and local limit theorem for percolation clusters, Elec. J. Prob. 14 (2009), paper no. 1, 26 pp.

[14] Bass, R.F. and Kassmann, M.: Harnack inequalities for non-local operators of variable order, Trans. Amer. Math. Soc. 357 (2005), 837-850.

[15] Bass, R.F. and Levin, D.A.: Transition probabilities for symmetric jump processes, Trans. Amer. Math. Soc. 354 (2002), 2933-2953.

[16] Berger, N. and Biskup, M.: Quenched invariance principle for simple random walk on percolation clusters, Probab. Theory Relat. Fields 137 (2007), 83-120.

[17] Berger, N., Biskup, M., Hoffman, C.E. and Kozma, G.: Anomalous heat-kernel decay for random walk among bounded random conductances, Ann. Inst. Henri Poincaré Probab. Statist 44 (2008), 374-392.

[18] Billingsley, P.: Convergence of Probability Measures, Wiley series in probability and statistics, New York, Second Edition, 1999.

[19] Biskup, M.: Recent progress on the random conductance model, Probab. Surv. 8 (2011), 294-373.

[20] Biskup, M. and Prescott, T.-M.: Functional CLT for random walk among bounded random conductances, Electron. J. Probab. 12 (2007), no. 49, 1323-1348.

[21] Biskup, M. and Rodriguez, P.-F.: Limit theory for random walks in degenerate time-dependent random environments, J. Funct. Anal. 274 (2018), 985-1046.

[22] Boukhadra, O., Kumagai, T. and Mathieu, P.: Harnack inequalities and local central limit theorem for the polynomial lower tail random conductance model, J. Math. Soc. Japan 67 (2015), 1413-1448.

[23] Carlen, E.A., Kusuoka, S. and Stroock, D.W.: Upper bounds for symmetric Markov transition functions, Ann. Inst. Henri Poincaré, Section B 23 (1987), 245-287.

[24] Chen, X., Kumagai, T. and Wang, J.: Random conductance models with stable-like jumps I: quenched invariance principle, preprint 2018, available at arXiv:1805.04344.

[25] Chen, Z.-Q. and Kumagai, T.: Heat kernel estimates for stable-like processes on $d$-sets, Stoch. Proc. Appl. 108 (2003), 27-62.

[26] Chen, Z.-Q. and Kumagai, T.: Heat kernel estimates for jump processes of mixed types on metric measure spaces, Probab. Theory Related Fields 140 (2008), 277-317.

[27] Chen, Z.-Q., Kumagai, T. and Wang, J.: Stability of heat kernel estimates for symmetric non-local Dirichlet forms, to appear in Memoirs of the AMS

[28] Chen, Z.-Q., Kumagai, T. and Wang, J.: Stability of parabolic Harnack inequalities for symmetric non-local Dirichlet forms, to appear in J. European Math. Soc.

[29] Chen, Z.-Q., Kumagai, T. and Wang, J.: Elliptic Harnack inequalities for symmetric non-local Dirichlet forms, to appear in J. Math. Pures Appl.

[30] Chen, Z.-Q., Kim, P. and Kumagai, T.: Discrete approximation of symmetric jump processes on metric measure spaces, Probab. Theory Related Fields 155 (2013), 703-749.

[31] Coulhon, T. and Grigor'yan, A.: Random walks on graphs with regular volume growth, Geom. Funct. Anal. 8 (1998), 656-701.

[32] Crawford, N. and Sly, A.: Simple random walks on long range percolation cluster I: heat kernel bounds, Probab. Theory Relat. Fields 154 (2012), 753-786.

[33] Crawford, N. and Sly, A.: Simple random walks on long range percolation cluster II: scaling limit, Ann. Probab. 41 (2013), 445-502.

[34] Crodyon, D.A. and Hambly, B.M.: Local limit theorems for sequences of simple random walks on graphs, Potential Anal. 29 (2008), 351-389.

[35] Davies, E.B.: Large deviations for the heat kernels on graphs, J. London Math. Soc. 23 (1993), 65-72.

[36] Delmotte, T.: Parabolic Harnack inequality and estimates of Markov chains on graphs, Rev. Mat. Iberoam. 15 (1999), 181-232.

[37] Dyda, B. and Kassmann, M.: On weighted Poincaré inequalities, Ann. Acad. Sci. Fenn. Math. 38 (2013), 721-726.

[38] Dyda, B. and Kassmann, M.: Regularity estimates for elliptic nonlocal operators, preprint 2015, available at arXiv:1509.08320

[39] Felsinger, M. and Kassmann, M.: Local regularity for parabolic nonlocal operators, Comm. Partial Differential Equations 38 (2013), 1539-1573.

[40] Folz, M.: Gaussian upper bounds for heat kernels of continuous time simple random walks, Elect. J. Probab. 16 (2011), 1693-1722. 
[41] Grigor'yan, A.: Gaussian upper bounds for heat kernel on arbitrary manifolds, J. Differ. Geom. 45 (1997), 33-52.

[42] Grigor'yan, A., Hu, E. and Hu, J.: Lower estimates of heat kernels for non-local Dirichlet forms on metric measure spaces, J. Funct. Anal. 272 (2017), 3311-3346.

[43] Grigor'yan, A., Hu, E. and Hu, J.: Two-sided estimates of heat kernels of jump type Dirichlet forms, Advances in Math. 330 (2018), 433-515.

[44] Grimmett, G.R.: Percolation, 2nd edition. Springer, Berlin, 1999.

[45] Ikeda, N. and Watanabe, S.: On some relations between the harmonic measure and the Lévy measure for a certain class of Markov processes, J. Math. Kyoto Univ. 2 (1962), 79-95.

[46] Kumagai, T.: Random walks on disordered media and their scaling limits, Lect. Notes in Math. 2101, Ecole d'été de probabilités de Saint-Flour XL-2010, Springer, New York 2014.

[47] Murugan, M. and Saloff-Coste, L.: Transition probability estimates for long range random walks, New York Journal of Mathematics 21 (2015), 723-757.

[48] Murugan, M. and Saloff-Coste, L.: Heat kernel estimates for anomalous heavy-tailed random walks, to appear in Ann. Inst. Henri Poincaré Probab. Statist.

[49] Mathieu, P. and Remy, E.: Isoperimetry and heat kernel decay on percolation clusters, Ann. Probab. 32 (2004), 100-128.

[50] Mathieu, P.: Quenched invariance principles for random walks with random conductances, J. Stat. Phys. 130 (2008), 1025-1046.

[51] Mathieu, P. and Piatnitski, A.: Quenched invariance principles for random walks on percolation clusters, Proc. Roy. Soc. A 463 (2007), 2287-2307.

[52] Procaccia, E., Rosenthal, R. and Sapozhnikov, A.: Quenched invariance principle for simple random walk on clusters in correlated percolation models, Probab. Theory Relat. Fields 166 (2016), 619-657.

[53] Sapozhnikov, A.: Random walks on infinite percolation clusters in models with long-range correlations, Ann. Probab. 45 (2017), 1842-1898.

[54] Sidoravicius, V. and Sznitman, A.-S.: Quenched invariance principles for walks on clusters of percolation or among random conductances, Probab. Theory Relat. Fields 129 (2004), 219-244. 